\title{
Harmonic functions on the branching graph associated with the infinite wreath product of a compact group
}

\author{
Akihito Hora and Takeshi Hirai
}

\begin{abstract}
A detailed study of the characters of $\mathfrak{S}_{\infty}(T)$, the wreath product of compact group $T$ with the infinite symmetric group $\mathfrak{S}_{\infty}$, is indispensable for harmonic analysis on this big group. In preceding works, we investigated limiting behavior of characters of the finite wreath product $\mathfrak{S}_{n}(T)$ as $n \rightarrow \infty$ and its connection with characters of $\mathfrak{S}_{\infty}(T)$. This paper takes a dual approach to these problems. We study harmonic functions on $\mathbb{Y}(\widehat{T})$, the branching graph of the inductive system of $\mathfrak{S}_{n}(T)$ 's, and give a classification of the minimal nonnegative harmonic functions on it. This immediately implies a classification of the characters of $\mathfrak{S}_{\infty}(T)$, which is a logically independent proof of the one obtained in earlier works. We obtain explicit formulas for minimal nonnegative harmonic functions on $\mathbb{Y}(\widehat{T})$ and Martin integral expressions for harmonic functions.
\end{abstract}

\section{Introduction}

Let $\mathfrak{S}_{n}(T)$ and $\mathfrak{S}_{\infty}(T)$ denote the wreath products of a compact group $T$ with the symmetric group $\mathfrak{S}_{n}$ of the $n$th degree and the infinite symmetric group $\mathfrak{S}_{\infty}$, respectively. The present paper constitutes a part of our project on harmonic analysis of $\mathfrak{S}_{\infty}(T)$. It is closely related to our previous works [7] and [9] published jointly with E. Hirai, in which asymptotic behavior of characters of $\mathfrak{S}_{n}(T)$ and its connection with characters of $\mathfrak{S}_{\infty}(T)$ are analyzed in detail from different points of view. The present paper thus cuts a clearer figure when it is put together with [7] and [9]. However, we keep it self-contained and feature independent results and approach. Let us explain the problems we treat as well as how they are related to [7] and [9]. Several notions freely used in this section are defined and explained in appropriate places of later sections.

Let $D_{\infty}(T)$ denote the restricted direct product of $T$. Then, $\mathfrak{S}_{\infty}$ canonically acts on $D_{\infty}(T)$ (see (1.13) and (1.14)), and $\mathfrak{S}_{\infty}(T)$ is the semidirect product of $D_{\infty}(T)$ and $\mathfrak{S}_{\infty}$ under this action. We equip $\mathfrak{S}_{\infty}(T)$ with the inductive limit topology. The following problems are fundamental in the harmonic analysis of $\mathfrak{S}_{\infty}(T)$ :

(a) classification of the finite factorial (or primary) unitary representations;

Kyoto Journal of Mathematics, Vol. 54, No. 4 (2014), 775-817

DOI 10.1215/21562261-2801822, (C) 2014 by Kyoto University

Received December 4, 2012. Revised June 10, 2013. Accepted September 27, 2013.

2010 Mathematics Subject Classification: Primary 20C32; Secondary 20P05, 20E22. 
(b) canonical direct integral decomposition of a finite unitary representation into factorial ones.

One knows that (b) is solved in an abstract sense as the central decomposition by virtue of von Neumann's reduction theory. We then get a measure for the superposition on the classification space of (a). Concrete computation of this measure remains as an independent and difficult problem. Concerning (a), we recall that the following four objects are equivalent. In other words, there exists a bijective correspondence between one another:

(a.1) the quasiequivalence classes of finite factorial unitary representations of $\mathfrak{S}_{\infty}(T)$;

(a.2) the extremal points of

$$
\mathcal{K}\left(\mathfrak{S}_{\infty}(T)\right)=\left\{f: \mathfrak{S}_{\infty}(T) \longrightarrow \mathbb{C} \mid\right.
$$

$f$ is continuous, positive definite, central, and normalized $\}$;

(a.3) the extremal points of

$$
\begin{aligned}
\mathcal{H}(\mathbb{Y}(\widehat{T}))= & \{\varphi: \mathbb{Y}(\widehat{T}) \longrightarrow \mathbb{C} \mid \\
& \varphi \text { is harmonic, nonnegative, and normalized }\}
\end{aligned}
$$

(a.4) the extremal points of

$$
\mathcal{M}(\mathfrak{T}(\widehat{T}))=\{\text { central probabilities on } \mathfrak{T}(\widehat{T})\} .
$$

Let us explain the notation used above. Let $\mathbb{Y}(\widehat{T})$ denote the vertices of the branching graph for the $\mathfrak{S}_{n}(T)$ 's (see (1.16)). The harmonicity of $\varphi$ is defined in (1.18). The term "normalized" means that $f(e)=1$ in $(0.1)$ and $\varphi(\varnothing)=1$ in (0.2). Let $\mathfrak{T}(\widehat{T})$ denote the set of infinite paths from $\varnothing$ on the branching graph. The centrality of a measure in (0.3) is defined in (3.1). The correspondence between (a.1) and (a.2) is described in [3]. Explicit realizations of finite factorial unitary representations of $\mathfrak{S}_{\infty}(T)$ in terms of the classifying parameters are given in [6]. We refer to [9] for the bijections between (0.1), (0.2), and (0.3). Later in Section 4, we review these bijections in a slightly wider context, namely, in the case of a general inductive system of compact groups. Furthermore, relations between their topologies are also discussed. An extremal point of (0.1) is called a character of $\mathfrak{S}_{\infty}(T)$. The second author and E. Hirai published several papers on the determination of the set of all characters of $\mathfrak{S}_{\infty}(T)$. A final form is given in [4] and [5]. The main purpose of the series of works that includes [7], [9], and the present paper is to understand the above four objects (a.1)-(a.4) for $\mathfrak{S}_{\infty}(T)$ through limiting procedures from the inductive system of $\mathfrak{S}_{n}(T)$ 's and thereby to give a sufficiently concrete answer to the fundamental problem of (a). These three papers take different approaches and methods. The present paper takes an approach from the viewpoint of (a.3). In contrast with this, [7] and [9] have (a.2) and (a.4), respectively, as their main targets. In the case where $T$ is a finite group, such a character theory was developed in [2]. 
We give a characterization of (a.3) by computing the Martin boundary $\partial \mathbb{Y}(\widehat{T})$ of the branching graph $\mathbb{Y}(\widehat{T})$. This approach is strongly motivated by works treating the Young graph and its variations such as [17], [12], [14], [1], [13], and [15]. It turns out that the minimal Martin boundary $\partial_{m} \mathbb{Y}(\widehat{T})$ is a proper noncompact subset of $\partial \mathbb{Y}(\widehat{T})$ if and only if the compact group $T$ is continuous. As an alternative answer to problem (b), we show the Martin integral representation for any element $\varphi$ in (0.2) as

$$
\varphi(\Lambda)=\int_{\Delta} \varphi_{\omega}(\Lambda) Q(d \omega)
$$

in which $\Delta$ realizing $\partial_{m} \mathbb{Y}(\widehat{T})$ and the kernel function $\varphi_{\omega}(\Lambda)$ are explicitly given together with the manner of obtaining probability $Q$ from $\varphi$. In general, Choquet's theorem enables us to have an abstract framework in which any element of a compact convex subset of a locally convex space is expressed as an integral over the extremal points. While Choquet's theorem is quite powerful, the superposition measure (namely, probability $Q$ in (0.4)) is highly unclear since it is obtained in a transcendental manner. We prove (0.4) by using a constructive method as an analogue of the 'radial limit' for a harmonic function on the unit disk of $\mathbb{C}$ (see [12] for the Young graph), without relying on Choquet's theorem.

This paper is organized as follows. In Section 1, we review necessary facts about representations of wreath product groups $\mathfrak{S}_{n}(T)$ and their branching graph $\mathbb{Y}(\widehat{T})$. Irreducible character formulas for $\mathfrak{S}_{n}(T)$ are discussed in some detail since their asymptotic behavior is crucial for our purpose. In particular, we need to formulate the expressions from which we can read out the asymptotics as $n \rightarrow \infty$. The branching graph is constructed from the branching rule for $\mathfrak{S}_{n}(T)$ 's. We recall several notions in the theory of Markov chains on graphs. Since there may be some differences in the terminology of Martin boundaries between probabilistic contexts and representation-theoretical ones, we give a brief account in Appendix A. Section 2 is devoted to the computation of the Martin boundary of $\mathbb{Y}(\widehat{T})$ by using irreducible characters of $\mathfrak{S}_{n}(T)$. In Section 3 , we prove an integral representation formula for harmonic functions on $\mathbb{Y}(\widehat{T})$. Combining the integral representation with the results in Section 2, we obtain a complete characterization of the minimal Martin boundary of $\mathbb{Y}(\widehat{T})$. The aim of Section 4 is to explain the relations between (0.1)-(0.3) from a general viewpoint with emphasis on their topologies.

\section{Preliminaries about wreath product groups and branching graphs}

Throughout the present paper, let $T$ be a compact group with identity element $e_{T}$. Let $[T]$ and $\widehat{T}$ denote the set of conjugacy classes of $T$ and the set of equivalence classes of continuous irreducible unitary representations of $T$, respectively. The group $T$ may be noncommutative and continuous. For technical simplicity, however, we assume that $\widehat{T}$ is at most countable. In this section, we recall necessary notions and some known results on wreath products of a compact group 
and associated branching graphs. In what follows, continuous unitary representation is referred to as UR for short. Similarly, irreducible UR is abbreviated to IUR. For $n \in \mathbb{N}=\{1,2,3, \ldots\}, \mathfrak{S}_{n}$ denotes the symmetric group of degree $n$, and $\mathbb{Y}_{n}$ denotes the set of Young diagrams of size $n$. Set $\mathbb{Y}=\bigsqcup_{n=0}^{\infty} \mathbb{Y}_{n}$, the totality of all Young diagrams, where $\mathbb{Y}_{0}=\{\varnothing\}$. We use the following (conventional) notation and terminologies for Young diagrams. For $\lambda \in \mathbb{Y}_{n}$,

- $\lambda_{j}$ is the length of the $j$ th row where $\lambda_{1} \geqq \lambda_{2} \geqq \cdots$;

- $m_{i}(\lambda)$ is the number of rows of length $i$ contained in $\lambda$;

- $l(\lambda)=\sum_{i=1}^{\infty} m_{i}(\lambda)$ is the number of rows of $\lambda$;

- $|\lambda|=\sum_{j=1}^{l(\lambda)} \lambda_{j}=\sum_{i=1}^{\infty} i m_{i}(\lambda)=n$ is the size of $\lambda$;

- $\pi^{\lambda}$ is an IUR of $\mathfrak{S}_{n}$ labeled by $\lambda$;

- $\chi^{\lambda}=\operatorname{tr} \pi^{\lambda}$ is the associated irreducible character of $\mathfrak{S}_{n}$.

Note that we use 'tr' for the nonnormalized trace.

\subsection{Wreath product of $T$}

For $n \in \mathbb{N}, \mathfrak{S}_{n}$ canonically acts on $T^{n}$, the $n$-fold direct product of $T: \sigma \in \mathfrak{S}_{n}$, $\boldsymbol{t}=\left(t_{1}, \ldots, t_{n}\right) \in T^{n}$,

$$
\sigma(\boldsymbol{t})=\left(t_{\sigma^{-1}(1)}, \ldots, t_{\sigma^{-1}(n)}\right) .
$$

This action gives the semidirect product $T^{n} \rtimes \mathfrak{S}_{n}$, which is denoted by $\mathfrak{S}_{n}(T)$ and called the wreath product of $T$ with $\mathfrak{S}_{n}$. We have $\mathfrak{S}_{1}(T)=T$ from the definition, and set $\mathfrak{S}_{0}(T)=\{e\}$ for notational convenience.

\subsection{Standard decomposition into basic elements}

Any nontrivial element $g=(\boldsymbol{t}, \sigma)$ in $\mathfrak{S}_{n}(T)$, where $\boldsymbol{t} \in T^{n}$ and $\sigma \in \mathfrak{S}_{n}$, has the standard decomposition

$$
g=\xi_{q_{1}} \cdots \xi_{q_{r}} g_{1} \cdots g_{m}
$$

into two kinds of basic elements $\xi_{q_{i}}$ and $g_{j}$ as follows. For each $q \in\{1,2, \ldots, n\}$, $\xi_{q}$ in (1.1) denotes an element in $T^{n}$ with nontrivial $t_{q} \neq e_{T}$ only at the $q$ th entry:

$$
\xi_{q}=\left(e_{T}, \ldots, e_{T}, t_{q}, e_{T}, \ldots, e_{T}\right), \quad t_{q} \in T,
$$

where $\{q\}$ is referred to as $\operatorname{supp} \xi_{q}$ (= support of $\xi_{q}$ ). We use the notation as $\xi_{q}=\left(t_{q},(q)\right)$ for $\xi_{q}$ above. Each $g_{j}$ in (1.1) has the form $\left(\boldsymbol{t}_{j}, \sigma_{j}\right)$ in which $\sigma_{j}$ is a cycle permutation in $\mathfrak{S}_{n}$ and $\boldsymbol{t}_{j}$ holds possibly nontrivial elements in $T$ only at the positions of $\operatorname{supp} \sigma_{j}$. Here $\operatorname{supp} \sigma_{j}$ denotes the set of letters in $\{1,2, \ldots, n\}$ of which the cycle $\sigma_{j}$ consists. Set $\operatorname{supp} g_{j}=\operatorname{supp} \sigma_{j}$. Moreover, all the supports

$$
\operatorname{supp} \xi_{q_{1}}, \ldots, \operatorname{supp} \xi_{q_{r}}, \operatorname{supp} g_{1}, \ldots, \operatorname{supp} g_{m}
$$

are taken to be disjoint in (1.1). Since $g=(\boldsymbol{t}, \sigma)$ is nontrivial in $\mathfrak{S}_{n}(T)$, the union of these supports is nonempty. It follows from (1.1) that $\sigma=\sigma_{1} \cdots \sigma_{m}$ is a cycle decomposition in $\mathfrak{S}_{n}$. The standard decomposition of $g$ in (1.1) is uniquely determined up to the orders of $\xi_{q_{i}}$ 's and $g_{j}$ 's. 


\subsection{The conjugacy classes of $\mathfrak{S}_{n}(T)$}

It is immediate that

$$
\sigma(t,(q)) \sigma^{-1}=(t, \sigma(q))
$$

for $\sigma \in \mathfrak{S}_{n}, q \in\{1,2, \ldots, n\}, t \in T$. For the basic element $(\boldsymbol{t}, \sigma), \sigma=\left(i_{1} i_{2} \cdots i_{l}\right)$, let $\boldsymbol{t}$ hold $t_{i_{j}} \in T$ at the position $i_{j}$, that is,

$$
\boldsymbol{t}=\prod_{j=1}^{l}\left(t_{i_{j}},\left(i_{j}\right)\right)
$$

Using (1.2) also, we have

$$
\begin{aligned}
\left(t_{i_{1}},\left(i_{1}\right)\right)^{-1} \boldsymbol{t} \sigma\left(t_{i_{1}},\left(i_{1}\right)\right) & =\left(t_{i_{1}}^{-1},\left(i_{1}\right)\right) \boldsymbol{t} \sigma\left(t_{i_{1}},\left(i_{1}\right)\right) \sigma^{-1} \sigma \\
& =\left(\prod_{j=2}^{l}\left(t_{i_{j}},\left(i_{j}\right)\right)\right)\left(t_{i_{1}},\left(i_{2}\right)\right) \sigma \\
& =\left(t_{i_{2}} t_{i_{1}},\left(i_{2}\right)\right)\left(\prod_{j=3}^{l}\left(t_{i_{j}},\left(i_{j}\right)\right)\right) \sigma .
\end{aligned}
$$

Repeating these conjugations, we see that $(\boldsymbol{t}, \sigma)$ is conjugate to

$$
\left(\left(t_{i_{l}} t_{i_{l-1}} \cdots t_{i_{2}} t_{i_{1}},\left(i_{l}\right)\right),\left(i_{1} i_{2} \cdots i_{l-1} i_{l}\right)\right) .
$$

Moreover, in (1.3), conjugacy class $\left[t_{i_{l}} \cdots t_{i_{2}} t_{i_{1}}\right]$ of $T$ is well defined since it is independent of the cyclic order of $t_{i_{1}}, \ldots, t_{i_{l}}$. A conjugacy class of $T$ and a 1-row Young diagram (corresponding to the conjugacy class of $l$-cycles in $\mathfrak{S}_{n}$ ) are thus assigned to the basic element $(\boldsymbol{t}, \sigma)$. In the expression of (1.1), we can add the product of

$$
\left(e_{T},(q)\right), \quad q \notin \bigsqcup_{i=1}^{r} \operatorname{supp} \xi_{q_{i}} \sqcup \bigsqcup_{j=1}^{m} \operatorname{supp} g_{j},
$$

without affecting anything. Furthermore, this yields the expression (1.1) even for the identity element $e$ in $\mathfrak{S}_{n}(T)$. We hence see that the conjugacy classes of $\mathfrak{S}_{n}(T)$ are parameterized by

$$
\mathbb{Y}_{n}([T])=\left\{\mathrm{P}=\left(\rho_{\theta}\right)_{\theta \in[T]}\left|\rho_{\theta} \in \mathbb{Y}, \sum_{\theta \in[T]}\right| \rho_{\theta} \mid=n\right\} .
$$

Let $C_{\mathrm{P}}$ denote the conjugacy class of $\mathfrak{S}_{n}(T)$ labeled by $\mathrm{P} \in \mathbb{Y}_{n}([T])$.

\subsection{The equivalence classes of IURs of $\mathfrak{S}_{n}(T)$}

By virtue of a standard inducing-up method, the equivalence classes of IURs of $\mathfrak{S}_{n}(T)$ are parameterized by

$$
\mathbb{Y}_{n}(\widehat{T})=\left\{\Lambda=\left(\lambda^{\zeta}\right)_{\zeta \in \widehat{T}}\left|\lambda^{\zeta} \in \mathbb{Y}, \sum_{\zeta \in \widehat{T}}\right| \lambda^{\zeta} \mid=n\right\} .
$$

In fact, IUR $\pi^{\Lambda}$ of $\mathfrak{S}_{n}(T)$ labeled by $\Lambda=\left(\lambda^{\zeta}\right)_{\zeta \in \widehat{T}} \in \mathbb{Y}_{n}(\widehat{T})$ is constructed as shown in [7, Section 3] (see also [9, Section 1.1]). For the case where $T$ is finite, 
we refer also to $[11$, Chapter 4]. To recall the construction, picking a partition of $\{1,2, \ldots, n\}$ into $\left|\lambda^{\zeta}\right|$-blocks:

$$
\{1,2, \ldots, n\}=\bigsqcup_{\zeta \in \widehat{T}} I_{n, \zeta}, \quad\left|I_{n, \zeta}\right|=\left|\lambda^{\zeta}\right|,
$$

we define IUR $\eta$ of $T^{n}$ by

$$
\eta=\bigotimes_{\zeta \in \widehat{T}}\left(\bigotimes_{i \in I_{n, \zeta}} \pi^{\zeta_{i}}\right), \quad \pi^{\zeta_{i}} \in \zeta_{i} \in \widehat{T}, \quad \zeta_{i} \equiv \zeta\left(i \in I_{n, \zeta}\right) .
$$

Here $\otimes$ denotes the outer tensor product of representations. We note, however, that the expression may not reflect the order of outer tensor products for simplicity of the notation. To be precise, taking distinct $\zeta^{\prime}, \zeta^{\prime \prime}, \zeta^{\prime \prime \prime} \in \widehat{T}$, let

$$
\Lambda=\left(\lambda^{\zeta}\right)_{\zeta \in \widehat{T}} \in \mathbb{Y}_{5}(\widehat{T}), \quad \lambda^{\zeta}= \begin{cases}(2), & \zeta=\zeta^{\prime}, \\ (1,1), & \zeta=\zeta^{\prime \prime} \\ (1), & \zeta=\zeta^{\prime \prime \prime} \\ \varnothing, & \text { otherwise, }\end{cases}
$$

for example. Assume that we take a partition of $\{1,2,3,4,5\}$ as

$$
\{1,4\} \sqcup\{2,5\} \sqcup\{3\}, \quad I_{5, \zeta^{\prime}}=\{1,4\}, \quad I_{5, \zeta^{\prime \prime}}=\{2,5\}, \quad I_{5, \zeta^{\prime \prime \prime}}=\{3\} .
$$

Then, we have $\eta=\pi^{\zeta^{\prime}} \otimes \pi^{\zeta^{\prime \prime}} \otimes \pi^{\zeta^{\prime \prime \prime}} \otimes \pi^{\zeta^{\prime}} \otimes \pi^{\zeta^{\prime \prime}}$ by definition. Under the action of $\sigma \in \mathfrak{S}_{n}$ on $\widehat{T^{n}}$ by

$$
\sigma \eta(\boldsymbol{t})=\eta\left(\sigma^{-1}(\boldsymbol{t})\right), \quad \sigma^{-1}(\boldsymbol{t})=\left(t_{\sigma(i)}\right)\left(\boldsymbol{t}=\left(t_{i}\right) \in T^{n}\right),
$$

the stationary subgroup $S^{\eta}=\left\{\sigma \in \mathfrak{S}_{n} \mid \sigma \eta \cong \eta\right\}$ is isomorphic to $\prod_{\zeta \in \widehat{T}} \mathfrak{S}_{I_{n, \zeta}} \cong$ $\prod_{\zeta \in \widehat{T}} \mathfrak{S}_{\left|\lambda^{\zeta}\right|}$. Set

$$
H_{n}=T^{n} \rtimes S^{\eta} \cong \prod_{\zeta \in \widehat{T}} \mathfrak{S}_{|\lambda \zeta|}(T) .
$$

Define IUR $\rho^{\zeta}$ of $\mathfrak{S}_{|\lambda \zeta|}(T)$ by

$$
\rho^{\zeta}(\boldsymbol{t}, \sigma)=\left(\pi^{\zeta}\right)^{\bigotimes\left|\lambda^{\zeta}\right|}(\boldsymbol{t}) I(\sigma) \quad\left(\boldsymbol{t} \in T^{\left|\lambda^{\zeta}\right|}, \sigma \in \mathfrak{S}_{|\lambda \zeta|}\right),
$$

where $I(\sigma)\left(\bigotimes_{i} v_{i}\right)=\bigotimes_{i} v_{\sigma^{-1}(i)}$ on $\left(V^{\zeta}\right)^{\otimes\left|\lambda^{\zeta}\right|}$, the representation space of $\left(\pi^{\zeta}\right)^{\bigotimes\left|\lambda^{\zeta}\right|}$. The $\rho^{\zeta}$ 's give an IUR of $H_{n}$ on the representation space of $\eta, V^{\eta} \cong$ $\bigotimes_{\zeta \in \widehat{T}}\left(V^{\zeta}\right)^{\otimes\left|\lambda^{\zeta}\right|}$, as $\rho^{\eta}=\bigotimes_{\zeta \in \widehat{T}} \rho^{\zeta}$. On the other hand, we have $\xi^{\eta}=\bigotimes_{\zeta \in \widehat{T}} \pi^{\lambda^{\zeta}}$ as an IUR of $S^{\eta}$, where $\xi^{\eta}$ is regarded as an IUR of $H_{n}$ by considering trivial actions of $T^{n}$. We see that $\rho^{\eta} \otimes \xi^{\eta}$ is an IUR of $H_{n}$. Then, $\pi^{\Lambda}$ is given by the induced representation

$$
\pi^{\Lambda}=\operatorname{Ind}_{H_{n}}^{\mathfrak{S}_{n}(T)} \rho^{\eta} \otimes \xi^{\eta}
$$

\subsection{Branching rule for $\mathfrak{S}_{n}(T)$ 's}

If $k<n$, then canonical inclusion $\iota_{n, k}: \mathfrak{S}_{k}(T) \longrightarrow \mathfrak{S}_{n}(T)$ is defined as $\iota_{n, k}(\boldsymbol{t}, \sigma)=$ $(\tilde{\boldsymbol{t}}, \tilde{\sigma})$, where

$$
\tilde{\boldsymbol{t}}=\left(\boldsymbol{t}, e_{T}, \ldots, e_{T}\right) \in T^{k} \times T^{n-k}=T^{n}, \quad \tilde{\sigma}=\sigma(k+1)(k+2) \cdots(n) \in \mathfrak{S}_{n} .
$$


Under the inclusion $\iota_{n, k}, \mathfrak{S}_{k}(T)$ is regarded as a subgroup of $\mathfrak{S}_{n}(T)$. If $\Lambda \in \mathbb{Y}_{n}(\widehat{T})$ is obtained by adding a box at an entry of $\mathrm{M} \in \mathbb{Y}_{n-1}(\widehat{T})$, we say that $\Lambda$ is adjacent to $\mathrm{M}$ and write $\mathrm{M} \nearrow \Lambda$. In this situation, since the entry of $\mathrm{M}$ at which a box is put is uniquely determined, we denote it by $\zeta_{\mathrm{M}, \Lambda} \in \widehat{T}$. For $\Lambda \in \mathbb{Y}_{n}(\widehat{T})$ and the corresponding IUR $\pi^{\Lambda}$ of $\mathfrak{S}_{n}(T)$, the restriction of $\pi^{\Lambda}$ to subgroup $\mathfrak{S}_{n-1}(T)$, denoted by $\operatorname{Res}_{\mathfrak{S}_{n-1}(T)}^{\mathfrak{S}_{n}(T)} \pi^{\Lambda}$, has the following irreducible decomposition:

$$
\operatorname{Res}_{\mathfrak{S}_{n-1}(T)}^{\mathfrak{S}_{n}(T)} \pi^{\Lambda} \cong \bigoplus_{\mathrm{M} \in \mathbb{Y}_{n-1}(\widehat{T}): \mathrm{M} \nearrow \Lambda}\left[\operatorname{dim} \zeta_{\mathrm{M}, \Lambda}\right] \pi^{\mathrm{M}} .
$$

\subsection{Irreducible character formula for $\mathfrak{S}_{n}(T)$}

For $\Lambda \in \mathbb{Y}_{n}(\widehat{T})$, let

$$
\chi^{\Lambda}(g)=\operatorname{tr} \pi^{\Lambda}(g), \quad g \in \mathfrak{S}_{n}(T),
$$

be the character value of IUR $\pi^{\Lambda}$ at $g$. A formula for computing the value of (1.7) was given in [7, Theorem 4.5] (see also [9, Section 1.2]). For the purpose of this paper, we need to know the asymptotic behavior of irreducible characters of $\mathfrak{S}_{n}(T)$ along with a fixed conjugacy class type and growing IURs. Taking this situation into account, we recall the formula as follows. Let $g \in \mathfrak{S}_{k}(T)$ have the standard decomposition like (1.1), where $\xi_{q}$ may be $\left(e_{T},(q)\right)$. If $n \geqq k$, then $g$ considered as an element of $\mathfrak{S}_{n}(T)$ under the inclusion $\iota_{n, k}: \mathfrak{S}_{k}(T) \longrightarrow \mathfrak{S}_{n}(T)$ has the same standard decomposition as (1.1). As shown in [7, Theorem 4.5], we have, for $\Lambda \in \mathbb{Y}_{n}(\widehat{T})$,

$$
\begin{aligned}
\chi^{\Lambda}(g)= & \sum_{\mathcal{Q}, \mathcal{J}} \frac{\left(n-|Q|-\sum_{j \in J}\left|\sigma_{j}\right|\right) !}{\prod_{\zeta \in \widehat{T}}\left(\left|\lambda^{\zeta}\right|-\left|Q_{\zeta}\right|-\sum_{j \in J_{\zeta}}\left|\sigma_{j}\right|\right) !} \prod_{\zeta \in \widehat{T}}\left\{(\operatorname{dim} \zeta)^{\left|\lambda^{\zeta}\right|-\left|Q_{\zeta}\right|-\sum_{j \in J_{\zeta}}\left|\sigma_{j}\right|}\right. \\
& \left.\times\left(\prod_{q \in Q_{\zeta}} \chi^{\zeta}\left(t_{q}\right)\right)\left(\prod_{j \in J_{\zeta}} \chi^{\zeta}\left(P_{\sigma_{j}}\left(\boldsymbol{t}_{j}\right)\right)\right) \chi_{\left(\left|\sigma_{j}\right|\right)_{j \in J_{\zeta}} \sqcup\left(1^{\zeta \zeta\left|-\sum_{j \in J_{\zeta}}\right| \sigma_{j} \mid}\right)}\right\},
\end{aligned}
$$

where $g=(\boldsymbol{t}, \sigma), \sigma=\sigma_{1} \cdots \sigma_{m}, g_{j}=\left(\boldsymbol{t}_{j}, \sigma_{j}\right)(j \in\{1,2, \ldots, m\}),\left|\sigma_{j}\right|=\left|\operatorname{supp} \sigma_{j}\right|$,

$$
P_{\sigma_{j}}\left(\boldsymbol{t}_{j}\right)=t_{i_{l}} t_{i_{l-1}} \cdots t_{i_{1}}, \quad \sigma_{j}=\left(i_{1} i_{2} \cdots i_{l}\right), \boldsymbol{t}_{j}=\left(t_{i}\right)_{i \in \operatorname{supp} \sigma_{j}}
$$

$\chi^{\zeta}=\operatorname{tr} \pi^{\zeta}$ (irreducible character) for $\zeta \in \widehat{T},\left(\left|\sigma_{j}\right|\right)_{j \in J_{\zeta}}$ is a Young diagram of size $\sum_{j \in J_{\zeta}}\left|\sigma_{j}\right|$, and $\mathcal{Q}=\left(Q_{\zeta}\right)_{\zeta \in \widehat{T}}$ and $\mathcal{J}=\left(J_{\zeta}\right)_{\zeta \in \widehat{T}}$ are partitions of $Q=\left\{q_{1}, \ldots, q_{r}\right\}$ and $J=\{1,2, \ldots, m\}$, respectively. We note that (1.8) is valid either if $\xi_{q}$ might be $\left(e_{T},(q)\right)$ in the standard decomposition of $g \in \mathfrak{S}_{k}(T)$ or if not. In other words, we can take either of the following (i) or (ii) in the expression of the right-hand side of (1.8):

(i) $Q=\left\{q_{1}, q_{2}, \ldots, q_{r}\right\}$, where $t_{q_{i}} \neq e_{T}(i=1,2, \ldots, r)$,

(ii) $Q=\{1,2, \ldots, k\} \backslash \bigsqcup_{j=1}^{m} \operatorname{supp} \sigma_{j}$.

The verification reduces to the obvious identity: for $a, b \in \mathbb{N}$ and $a_{i} \in \mathbb{N} \cup\{0\}$ $(i=1, \ldots, p)$ such that $b \leqq a$ and $a_{1}+\cdots+a_{p}=a$, 


$$
\frac{a !}{a_{1} ! \cdots a_{p} !}=\sum_{\Delta=\left(\Delta_{i}\right)_{i=1}^{p}: \text { partition of }\{1, \ldots, b\}} \frac{(a-b) !}{\left(a_{1}-\left|\Delta_{1}\right|\right) ! \cdots\left(a_{p}-\left|\Delta_{p}\right|\right) !}
$$

with the convention $1 /(-n) !=0$ if $a_{i}<\left|\Delta_{i}\right|$. (Imagine dividing an $a$-set consisting of $b$ whites and $a-b$ blacks into $a_{1}$-set, $\ldots, a_{p}$-set.) The formula (1.8) can be expressed also by using the notation for a conjugacy class of $\mathfrak{S}_{k}(T)$ as (1.4). Let $g \in C_{\mathrm{P}} \subset \mathfrak{S}_{k}(T)$, where $\mathrm{P}=\left(\rho_{\theta}\right)_{\theta \in[T]}$. Picking up nontrivial $\rho_{\theta}$ 's (i.e., not equal to $\varnothing$ ), we write $\mathrm{P}=\left(\rho_{\theta_{i}}\right)_{i=1, \ldots, l}$. Then the standard decomposition (1.1) of $g$ has the form of

$$
g=\xi_{q_{1}^{\left(\theta_{1}\right)}} \cdots \xi_{q_{r\left(\theta_{1}\right)}^{\left(\theta_{1}\right)}} g_{1}^{\left(\theta_{1}\right)} \cdots g_{m\left(\theta_{1}\right)}^{\left(\theta_{1}\right)} \cdots \xi_{q_{1}^{\left(\theta_{l}\right)}} \cdots \xi_{q_{r\left(\theta_{l}\right)}^{\left(\theta_{l}\right)}} g_{1}^{\left(\theta_{l}\right)} \cdots g_{m\left(\theta_{l}\right)}^{\left(\theta_{l}\right)}
$$

where $r\left(\theta_{i}\right)=m_{1}\left(\rho_{\theta_{i}}\right), m\left(\theta_{i}\right)=m_{2}\left(\rho_{\theta_{i}}\right)+m_{3}\left(\rho_{\theta_{i}}\right)+\cdots$, and

$$
\xi_{q_{h}^{\left(\theta_{i}\right)}}=\left(t_{q_{h}^{\left(\theta_{i}\right)}},\left(q_{h}^{\left(\theta_{i}\right)}\right)\right), \quad g_{j}^{\left(\theta_{i}\right)}=\left(\boldsymbol{t}_{j}^{\left(\theta_{i}\right)}, \sigma_{j}^{\left(\theta_{i}\right)}\right), \quad \theta_{i}=\left[t_{q_{h}^{\left(\theta_{i}\right)}}\right]=\left[P_{\sigma_{j}^{\left(\theta_{i}\right)}}\left(\boldsymbol{t}_{j}^{\left(\theta_{i}\right)}\right)\right]
$$

for $h \in\left\{1,2, \ldots, r\left(\theta_{i}\right)\right\}, j \in\left\{1,2, \ldots, m\left(\theta_{i}\right)\right\}$. Note that

$$
k=\sum_{\theta \in[T]}\left|\rho_{\theta}\right|=\sum_{i=1}^{l}\left(r\left(\theta_{i}\right)+\sum_{j=1}^{m\left(\theta_{i}\right)}\left|\sigma_{j}^{\left(\theta_{i}\right)}\right|\right) .
$$

Let $\left(\rho_{\theta_{i}}\right)_{j}^{*}$ denote the $j$ th row of $\rho_{\theta_{i}}$. The length of $\left(\rho_{\theta_{i}}\right)_{j}^{*}$ is $\left(\rho_{\theta_{i}}\right)_{j}$. We distinguish $\left(\rho_{\theta_{i}}\right)_{j}^{*}$ from $\left(\rho_{\theta_{i^{\prime}}}\right)_{j^{\prime}}^{*}$ even if they have the same length (i.e., if $\left.\left(\rho_{\theta_{i}}\right)_{j}=\left(\rho_{\theta_{i^{\prime}}}\right)_{j^{\prime}}\right)$. Decomposing each $\rho_{\theta_{i}}$ into rows, consider the set of the rows of $\mathrm{P}$ :

$$
\operatorname{rows}(\mathrm{P})=\left\{\left(\rho_{\theta_{1}}\right)_{1}^{*}, \ldots,\left(\rho_{\theta_{1}}\right)_{l\left(\rho_{\theta_{1}}\right)}^{*}, \ldots,\left(\rho_{\theta_{l}}\right)_{1}^{*}, \ldots,\left(\rho_{\theta_{l}}\right)_{l\left(\rho_{\theta_{l}}\right)}^{*}\right\} .
$$

When (1.8) is applied to $g \in \mathfrak{S}_{k}(T)$ decomposed as $(1.9), \mathcal{Q}=\left(Q_{\zeta}\right)_{\zeta \in \widehat{T}}$ and $\mathcal{J}=$ $\left(J_{\zeta}\right)_{\zeta \in \widehat{T}}$ are partitions of

$$
\begin{aligned}
& \left\{q_{1}^{\left(\theta_{1}\right)}, \ldots, q_{r\left(\theta_{1}\right)}^{\left(\theta_{1}\right)}, \ldots, q_{1}^{\left(\theta_{l}\right)}, \ldots, q_{r\left(\theta_{l}\right)}^{\left(\theta_{l}\right)}\right\} \quad \text { and } \\
& \left\{g_{1}^{\left(\theta_{1}\right)}, \ldots, g_{m\left(\theta_{1}\right)}^{\left(\theta_{1}\right)}, \ldots, g_{1}^{\left(\theta_{l}\right)}, \ldots, g_{m\left(\theta_{l}\right)}^{\left(\theta_{l}\right)}\right\}
\end{aligned}
$$

respectively. It is immediate that there exists a bijective correspondence between

$$
\text { partition }(\mathcal{Q}, \mathcal{J}) \longleftrightarrow \operatorname{map}: \operatorname{rows}(\mathrm{P}) \longrightarrow \widehat{T} \text {. }
$$

In other words, partition $(\mathcal{Q}, \mathcal{J})$ is equivalent to giving the rows of $\mathrm{P}$ a $\widehat{T}$-labeling. Since $\pi^{\Lambda}$ (where $\Lambda=\left(\lambda^{\zeta}\right)_{\zeta \in \widehat{T}} \in \mathbb{Y}_{n}(\widehat{T})$ ) is given as an induced representation from subgroup $H_{n}=T^{n} \rtimes \prod_{\zeta \in \widehat{T}} \mathfrak{S}_{\left|\lambda^{\zeta}\right|}$ of $\mathfrak{S}_{n}(T), \chi^{\Lambda}(g)$ is nonzero only if $g$ is conjugate to an element of $H_{n}$. This condition for $g$ is rephrased in terms of conjugacy class $C_{\mathrm{P}}$ containing $g$ as: there exists a map $r: \operatorname{rows}(\mathrm{P}) \longrightarrow \widehat{T}$ such that

$$
\sum_{\left(\rho_{\theta_{i}}\right)_{j}^{*}: r\left(\left(\rho_{\theta_{i}}\right)_{j}^{*}\right)=\zeta}\left(\rho_{\theta_{i}}\right)_{j} \leqq\left|\lambda^{\zeta}\right|, \quad \zeta \in \widehat{T} .
$$

(Namely, the sum of the length of the rows labeled by $\zeta$ does not exceed $\left|\lambda^{\zeta}\right|$ for any $\zeta$.) In the case of $n=k,(1.11)$ is equivalent to the condition with $=$ replacing $\leqq$. The Young diagram consisting of the rows in $r^{-1}(\zeta)$ is denoted by the same notation $r^{-1}(\zeta)$. Similarly, the Young diagram consisting of the rows 
both in $r^{-1}(\zeta)$ and $\rho_{\theta}$ is denoted by $r^{-1}(\zeta) \cap \rho_{\theta}$. Under the correspondence of (1.10), we have

$$
\left(\left|\sigma_{j}\right|\right)_{j \in J_{\zeta}} \sqcup\left(1^{\left|Q_{\zeta}\right|}\right)=r^{-1}(\zeta), \quad \zeta \in \widehat{T} .
$$

Furthermore, we have, for $\zeta \in \widehat{T}$,

$$
\begin{aligned}
\left(\prod_{q \in Q_{\zeta}} \chi^{\zeta}\left(t_{q}\right)\right)\left(\prod_{j \in J_{\zeta}} \chi^{\zeta}\left(P_{\sigma_{j}}\left(\boldsymbol{t}_{j}\right)\right)\right) & =\prod_{\theta \in[T]}\left(\chi_{\theta}^{\zeta}\right)^{l\left(r^{-1}(\zeta) \cap \rho_{\theta}\right)}, \\
\chi_{\left(\left|\sigma_{j}\right|\right)_{j \in J_{\zeta}} \sqcup\left(1^{|\lambda \zeta|-\sum_{j \in J_{\zeta}}\left|\sigma_{j}\right|}\right)} & =\chi_{r^{-1}(\zeta) \sqcup\left(1|\lambda \zeta|-\left|r^{-1}(\zeta)\right|\right.}^{\lambda^{\zeta}} .
\end{aligned}
$$

Here a character is denoted by the notation $\chi$ with the upper and lower indices expressing the labels of a UR and a conjugacy class, respectively. Namely, $\chi_{\theta}^{\zeta}$ is the value at $\theta$ of the irreducible character $\chi^{\zeta}=\operatorname{tr} \pi^{\zeta}$ of $T$. In (1.8), we can assume that $|Q|+\sum_{j \in J}\left|\sigma_{j}\right|=k$ by putting elements of the form $\xi_{q}=\left(e_{T},(q)\right)$ in the standard decomposition of $g \in \mathfrak{S}_{k}(T)$ if necessary (see the note following (1.8)). For $\mathrm{P}=\left(\rho_{\theta}\right)_{\theta \in[T]} \in \mathbb{Y}_{k}([T])$, let us glue $n-k$ 1-box rows to $\rho_{\left\{e_{T}\right\}}$, the diagram at the $\left\{e_{T}\right\}$-entry of $\mathrm{P}$, and let $\iota_{n, k}(\mathrm{P})$ denote the resulting element of $\mathbb{Y}_{n}([T])$, that is, $\iota_{n, k}(\mathrm{P})=\left(\tilde{\rho}_{\theta}\right)_{\theta \in[T]}$, where

$$
\tilde{\rho}_{\theta}= \begin{cases}\rho_{\theta}, & \theta \neq\left\{e_{T}\right\}, \\ \rho_{\left\{e_{T}\right\}} \sqcup\left(1^{n-k}\right), & \theta=\left\{e_{T}\right\} .\end{cases}
$$

Then, we have that $C_{\iota_{n, k}(\mathrm{P})} \cap \mathfrak{S}_{k}(T)=C_{\mathrm{P}}$ under the inclusion $\mathfrak{S}_{k}(T) \subset \mathfrak{S}_{n}(T)$ by $\iota_{n, k}$. After these preparations, we get an alternative expression of (1.8) as

$$
\begin{aligned}
\chi_{\iota_{n, k}(\mathrm{P})}^{\Lambda}= & \sum_{r \text { satisfying }(1.11)} \frac{(n-k) !}{\prod_{\zeta \in \widehat{T}}\left(\left|\lambda^{\zeta}\right|-\left|r^{-1}(\zeta)\right|\right) !} \\
& \times \prod_{\zeta \in \widehat{T}}\left\{(\operatorname{dim} \zeta)^{\left|\lambda^{\zeta}\right|-\left|r^{-1}(\zeta)\right|}\right. \\
& \left.\times\left(\prod_{\theta \in[T]}\left(\chi_{\theta}^{\zeta}\right)^{l\left(r^{-1}(\zeta) \cap \rho_{\theta}\right)}\right) \chi_{r^{-1}(\zeta) \sqcup\left(1|\lambda \zeta|-\left|r^{-1}(\zeta)\right|\right)}^{\lambda^{\zeta}}\right\}
\end{aligned}
$$

for $\mathrm{P} \in \mathbb{Y}_{k}([T])$ and $\Lambda \in \mathbb{Y}_{n}(\widehat{T})$ with $k \leqq n$. If $k=n$, (1.12) has a simpler expression since $r$ satisfying (1.11) (necessarily $=$ instead of $\leqq$ ) yields $\left|\lambda^{\zeta}\right|=\left|r^{-1}(\zeta)\right|$ for any $\zeta \in \widehat{T}$.

\subsection{Infinite wreath product}

Here, $\mathfrak{S}_{\infty}$ denotes the infinite symmetric group, the set of all finite permutations of $\mathbb{N}$, and $\mathfrak{S}_{\infty}$ acts on the restricted direct product of $T$

$$
D_{\infty}(T)=\left\{\boldsymbol{t}=\left(t_{1}, t_{2}, \ldots\right) \in T^{\infty} \mid t_{j}=e_{T} \text { except finitely many } j \text { 's }\right\}
$$

canonically by

$$
\sigma(\boldsymbol{t})=\left(t_{\sigma^{-1}(1)}, t_{\sigma^{-1}(2)}, \ldots\right), \quad \sigma \in \mathfrak{S}_{\infty}, \boldsymbol{t} \in D_{\infty}(T) .
$$


The semidirect product $D_{\infty}(T) \rtimes \mathfrak{S}_{\infty}$ generated by this action is called the wreath product of $T$ with $\mathfrak{S}_{\infty}$ and is denoted by $\mathfrak{S}_{\infty}(T)$. The canonical inclusion $\iota_{n}$ : $\mathfrak{S}_{n}(T) \longrightarrow \mathfrak{S}_{\infty}(T)$ satisfies $\iota_{n} \circ \iota_{n, k}=\iota_{k}$ for $k<n$. Under this inclusion map, we regard $\mathfrak{S}_{\infty}(T)$ as $\bigcup_{n=0}^{\infty} \mathfrak{S}_{n}(T)$. Since the standard decomposition (1.1) is stable for $n$ 's large enough, we get the standard decomposition into basic elements for each element in $\mathfrak{S}_{\infty}(T)$. The conjugacy classes of $\mathfrak{S}_{\infty}(T)$ are then parameterized by

$$
\mathbb{Y}([T])=\left\{\mathrm{P}=\left(\rho_{\theta}\right)_{\theta \in[T]} \mid \rho_{\theta} \in \mathbb{Y}, m_{1}\left(\rho_{\left\{e_{T}\right\}}\right)=0\right\} .
$$

In (1.15), the trivial cycles are omitted; for example, the trivial conjugacy class $\{e\}$ of $\mathfrak{S}_{\infty}(T)$ corresponds to $\mathrm{P}=\left(\rho_{\theta}\right)$ with $\rho_{\theta}=\varnothing$ for any $\theta \in[T]$ (instead of $\left.\rho_{\left\{e_{T}\right\}}=\left(1^{\infty}\right)\right)$. Note that $\varnothing$ satisfies $m_{1}(\varnothing)=0$.

\subsection{Branching graph}

Considering $\mathbb{Y}_{n}(\widehat{T})$ in $(1.5)$, set

$$
\mathbb{Y}(\widehat{T})=\bigsqcup_{n=0}^{\infty} \mathbb{Y}_{n}(\widehat{T}),
$$

where $\mathbb{Y}_{0}(\widehat{T})$ is by definition the singleton set $\{\varnothing\}$. Note that $\mathbb{Y}_{1}(\widehat{T})=\widehat{T}$. Among the vertices $\mathbb{Y}(\widehat{T})$, let us define the edge structure by taking the branching rule (1.6) into account. Namely, $\Lambda \in \mathbb{Y}_{n}(\widehat{T})$ and $\mathrm{M} \in \mathbb{Y}_{n+1}(\widehat{T})$ are joined by an edge if and only if $\Lambda \nearrow \mathrm{M}$. Moreover, we put multiplicity

$$
\kappa(\Lambda, \mathrm{M})=\operatorname{dim} \zeta_{\Lambda, \mathrm{M}}
$$

on the edge $\Lambda \nearrow \mathrm{M}$.

\section{EXAMPLE 1.1}

Let $T$ be $\mathfrak{S}_{3}$, the symmetric group of degree $3 ; \widehat{\mathfrak{S}_{3}}$ consists of $\zeta_{1}$ (equal to 1 ), $\zeta_{2}$ (equal to $\operatorname{sgn}$ ), and $\zeta_{3}$ where $\operatorname{dim} \zeta_{3}=2$. According to this order, an element of $\mathbb{Y}\left(\widehat{\mathfrak{S}_{3}}\right)$ is expressed as $\left(\lambda^{1}, \lambda^{2}, \lambda^{3}\right)$ with $\lambda^{j} \in \mathbb{Y}$. The very beginning of the branching graph $\mathbb{Y}\left(\widehat{\mathfrak{S}_{3}}\right)$ is as drawn in Figure 1.

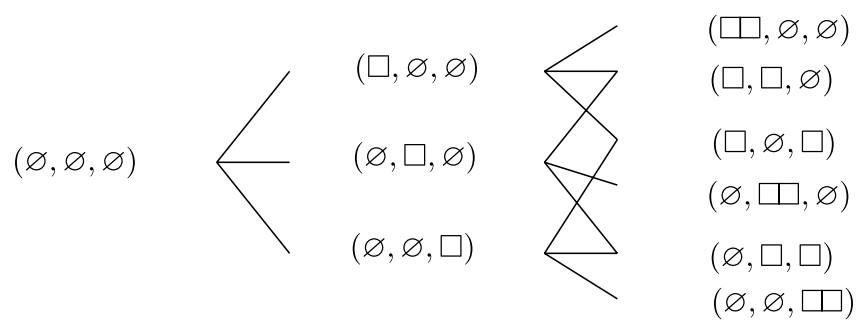

Figure 1. Branching graph $\mathbb{Y}\left(\widehat{\mathfrak{S}_{3}}\right)$. 


\subsection{Harmonic function}

Let us give the precise definition of $(0.2)$. A $\mathbb{C}$-valued function $\varphi$ on $\mathbb{Y}(\widehat{T})$ is said to be harmonic if

$$
\varphi(\Lambda)=\sum_{\mathrm{M}: \Lambda \nearrow \mathrm{M}} \kappa(\Lambda, \mathrm{M}) \varphi(\mathrm{M}), \quad \Lambda \in \mathbb{Y}(\widehat{T}),
$$

holds. If $\varphi(\varnothing)=1$ holds, then $\varphi$ is said to be normalized. Since (1.18) reflects directly the branching rule (1.6), or equivalently,

$$
\operatorname{Ind}_{\mathfrak{S}_{n}(T)}^{\mathfrak{S}_{n+1}(T)} \pi^{\Lambda} \cong \bigoplus_{\mathrm{M}: \Lambda \nearrow \mathrm{M}}\left[\operatorname{dim} \zeta_{\Lambda, \mathrm{M}}\right] \pi^{\mathrm{M}}
$$

defining harmonicity through (1.18) is canonical from the viewpoint of representation theory. On the other hand, (1.18) seems to be noncanonical from the viewpoint of probability theory or (discrete) potential theory since harmonicity is usually defined by using a transition probability as

$$
\varphi(\Lambda)=\sum_{\mathrm{M}} p(\Lambda, \mathrm{M}) \varphi(\mathrm{M})
$$

where $p(\Lambda, \mathrm{M}) \geqq 0$ and $\sum_{\mathrm{M}} p(\Lambda, \mathrm{M})=1$. For example, a constant function is not harmonic according to (1.18). We review basic definitions and necessary properties of the Martin boundary in Appendix A. As noted there, Martin boundary theories are equivalent based on either (1.18) or (1.20) by virtue of what is called the ' $h$-transform' method. Let us proceed with (1.18).

\subsection{Dimension function}

On the branching graph $\mathbb{Y}(\widehat{T})$, a path $u$ joining $\Lambda \in \mathbb{Y}_{l}(\widehat{T})$ to $\mathrm{M} \in \mathbb{Y}_{m}(\widehat{T})$, where $l<m$, is expressed as

$$
u=(u(l) \nearrow u(l+1) \nearrow \cdots \nearrow u(m-1) \nearrow u(m)), \quad u(l)=\Lambda, u(m)=\mathrm{M} .
$$

We set

$$
w_{u}=\prod_{i=l}^{m-1} \kappa(u(i), u(i+1)),
$$

and we call $w_{u}$ the weight of path $u$. Set also

$$
d(\Lambda, \mathrm{M})=\sum_{\text {path } u: \Lambda \nearrow \ldots \nearrow \mathrm{M}} w_{u},
$$

which means the number of weighted paths from $\Lambda$ to $\mathrm{M}$. In particular, set

$$
d(\Lambda)=d(\varnothing, \Lambda)=\operatorname{dim} \pi^{\Lambda}, \quad \Lambda \in \mathbb{Y}(\widehat{T}) .
$$

The second equality of (1.23) follows from iterating (1.6) and then using (1.22). The functions $d$ in (1.22) and (1.23) are called dimension functions on $\mathbb{Y}(\widehat{T})$. Although dimension functions are defined on a general branching through weights of paths as (1.22), we see that, in the case of branching graph $\mathbb{Y}(\widehat{T})$ for wreath product groups, weight $w_{u}$ in (1.21) depends only on the initial $\Lambda=\left(\lambda^{\zeta}\right)$ and 
terminal $\mathrm{M}=\left(\mu^{\zeta}\right)$, and is expressed as

$$
w_{u}=\prod_{\zeta \in \widehat{T}}(\operatorname{dim} \zeta)^{\left|\mu^{\zeta}\right|-\left|\lambda^{\zeta}\right|},
$$

which is directly seen from the branching rule (1.6). Hence (1.22) is reduced to the case of a simple (i.e., multiplicity-free) branching graph. Boyer [2] pointed out this phenomenon and applied it to develop character theory of the infinite wreath product of a finite group. According to the cardinality $|T|$ of compact group $T$, set

$$
T^{\natural}= \begin{cases}\mathbb{T}\left(\text { torus } \cong S^{1}\right), & |T|=\infty, \\ \mathbb{Z} / p \mathbb{Z}, & |T|=p<\infty .\end{cases}
$$

Here, $d^{\natural}$ denotes the dimension function on the branching graph for $\mathfrak{S}_{n}\left(T^{\natural}\right)^{\text {'s. }}$ Putting (1.24) into (1.22), we have

$$
\begin{aligned}
d(\Lambda, \mathrm{M}) & =\prod_{\zeta \in \widehat{T}}(\operatorname{dim} \zeta)^{\left|\mu^{\zeta}\right|-\left|\lambda^{\zeta}\right|} \sum_{\operatorname{path}} \sum_{u: \Lambda \nearrow \ldots \nearrow \mathrm{M}} 1 \\
& =\prod_{\zeta \in \widehat{T}}(\operatorname{dim} \zeta)^{\left|\mu^{\zeta}\right|-\left|\lambda^{\zeta}\right|} d^{\natural}(\Lambda, \mathrm{M}) .
\end{aligned}
$$

\subsection{Martin kernel}

We set

$$
K(\Lambda, \mathrm{M})=\frac{d(\Lambda, \mathrm{M})}{d(\mathrm{M})}, \quad \Lambda, \mathrm{M} \in \mathbb{Y}(\widehat{T})
$$

under the convention that $d(\Lambda, \mathrm{M})=0$ if there are no paths from $\Lambda$ to $\mathrm{M}$ on $\mathbb{Y}(\widehat{T})$. The equation in (1.27) agrees with a common terminology in Markov chain theory in which a Martin kernel is defined as a ratio of potential kernels (see Appen$\operatorname{dix}$ A). Letting $K^{\natural}$ denote the Martin kernel in accordance with (1.25) and (1.26) yields

$$
K(\Lambda, \mathrm{M})=\prod_{\zeta \in \widehat{T}} \frac{1}{(\operatorname{dim} \zeta)^{\lambda^{\zeta} \mid}} K^{\natural}(\Lambda, \mathrm{M}) .
$$

We introduce a distance on $\mathbb{Y}(\widehat{T})$ by

$$
D(\Lambda, \mathrm{M})=\sum_{\mathrm{N} \in \mathbb{Y}(\widehat{T})} C_{\mathrm{N}}\left(|K(\mathrm{~N}, \Lambda)-K(\mathrm{~N}, \mathrm{M})|+\left|\delta_{\mathrm{N}, \Lambda}-\delta_{\mathrm{N}, \mathrm{M}}\right|\right),
$$

$$
\Lambda, \mathrm{M} \in \mathbb{Y}(\widehat{T}),
$$

where $C_{\mathrm{N}}$ is an appropriately chosen positive coefficient. Then, as noted in Appendix A, the following conditions (i) and (ii) are equivalent for the sequence

$$
\left\{\mathrm{M}^{(n)}=\left(\mu^{(n) \zeta}\right)_{\zeta \in \widehat{T}}\right\}_{n \in \mathbb{N}}
$$

in $\mathbb{Y}(\widehat{T})$.

(i) $\left\{\mathrm{M}^{(n)}\right\}_{n \in \mathbb{N}}$ is a Cauchy sequence with respect to $D$. 
(ii) Either there exists $M \in \mathbb{Y}(\widehat{T})$ such that

$$
\mathrm{M}^{(n)}=\mathrm{M} \quad \text { for sufficiently large } n,
$$

or else

$$
\begin{aligned}
& \left|\mathrm{M}^{(n)}\right|=\sum_{\zeta \in \widehat{T}}\left|\mu^{(n) \zeta}\right| \underset{n \rightarrow \infty}{\longrightarrow} \infty \text { and } \\
& \left\{K\left(\Lambda, \mathrm{M}^{(n)}\right)\right\}_{n \in \mathbb{N}} \text { is a Cauchy sequence in } \mathbb{R} \text { for any } \Lambda \in \mathbb{Y}(\widehat{T}) .
\end{aligned}
$$

We obtain the Martin compactification $\overline{\mathbb{Y}(\widehat{T})}$ by taking the completion of $\mathbb{Y}(\widehat{T})$ with respect to the distance $D$. As seen from (1.30) and (1.31), $\mathbb{Y}(\widehat{T})$ is an open subset of $\overline{\mathbb{Y}(\widehat{T})}$ on which the relative topology is discrete.

\subsection{Martin boundary of $\mathbb{Y}(\widehat{T})$}

The compact set $\overline{\mathbb{Y}(\widehat{T})} \backslash \mathbb{Y}(\widehat{T})$ is called the Martin boundary of $\mathbb{Y}(\widehat{T})$ and is denoted by $\partial \mathbb{Y}(\widehat{T})$ in this paper. The Martin kernel $K$ in (1.27) is extended as a continuous function on $\mathbb{Y}(\widehat{T}) \times \overline{\mathbb{Y}(\widehat{T})}$ :

$$
K(\Lambda, \omega)=\lim _{n \rightarrow \infty} K\left(\Lambda, \mathrm{M}^{(n)}\right), \quad(\Lambda, \omega) \in \mathbb{Y}(\widehat{T}) \times \overline{\mathbb{Y}(\widehat{T})},
$$

along with $\mathrm{M}^{(n)} \in \mathbb{Y}(\widehat{T}) \underset{n \rightarrow \infty}{\longrightarrow} \omega \in \overline{\mathbb{Y}(\widehat{T})}$, which is again called a Martin kernel. We have

$$
K(\varnothing, \omega)=1, \quad K(\Lambda, \omega) \geqq 0 .
$$

Consider the minimal Martin boundary of $\mathbb{Y}(\widehat{T})$

$$
\begin{aligned}
\partial_{m} \mathbb{Y}(\widehat{T})= & \{\omega \in \partial \mathbb{Y}(\widehat{T}) \mid \\
& K(\cdot, \omega) \text { is a minimal harmonic function on } \mathbb{Y}(\widehat{T})\} .
\end{aligned}
$$

Note that the Martin kernel $K(\cdot, \omega)$ is not necessarily harmonic on $\mathbb{Y}(\widehat{T})$ for $\omega \in \partial \mathbb{Y}(\widehat{T})$ as we actually see later in the case of continuous $T$. Here a nonnegative harmonic function $\varphi$ is said to be minimal if harmonic $\psi$ such that $0 \leqq \psi \leqq \varphi$ is necessarily a constant multiple of $\varphi$. Under the definition of $(0.2), \varphi \in \mathcal{H}(\mathbb{Y}(\widehat{T}))$ is extremal if and only if it is minimal. In fact, extremality of $\varphi$ implies

$$
\begin{aligned}
& 0 \leqq \psi \leqq \varphi \Longrightarrow \varphi=\psi(\varnothing) \cdot \frac{\psi}{\psi(\varnothing)}+(1-\psi(\varnothing)) \frac{\varphi-\psi}{1-\psi(\varnothing)} \\
& \Longrightarrow \varphi=\frac{\psi}{\psi(\varnothing)} \Longleftrightarrow \psi=\psi(\varnothing) \varphi .
\end{aligned}
$$

Conversely, minimality of $\varphi$ implies that

$$
\begin{aligned}
\varphi & =\alpha \psi_{1}+(1-\alpha) \psi_{2}, \quad 0 \leqq \alpha \leqq 1, \psi_{1}, \psi_{2} \in \mathcal{H}(\mathbb{Y}(\widehat{T})) \\
& \Longrightarrow \alpha \psi_{1}=\mathrm{const} \cdot \varphi, \quad(1-\alpha) \psi_{2}=\mathrm{const} \cdot \varphi \\
& \Longrightarrow \psi_{1}=\psi_{2}=\varphi
\end{aligned}
$$


Comparing (0.2) and (1.35), we hence have that

$$
\left\{K(\cdot, \omega) \mid \omega \in \partial_{m} \mathbb{Y}(\widehat{T})\right\} \subset \text { the extremal points of } \mathcal{H}(\mathbb{Y}(\widehat{T})) .
$$

Actually, we will see that the equality holds (see Theorem 3.3).

\section{Computation of the Martin boundary of $\mathbb{Y}(\widehat{T})$}

In order to compute the Martin boundary of $\mathbb{Y}(\widehat{T})$, we need a formula for the Martin kernel $K(\Lambda, \mathrm{M})$ from which its asymptotic behavior can be read out efficiently. Let us set some further notation on characters of $\mathfrak{S}_{n}$. The normalized irreducible character of $\mathfrak{S}_{n}$ corresponding to $\lambda \in \mathbb{Y}_{n}$ is denoted by $\tilde{\chi}^{\lambda}=\chi^{\lambda} / \operatorname{dim} \lambda$. For $\rho, \lambda \in \mathbb{Y}$, set

$$
\Sigma_{\rho}(\lambda)= \begin{cases}|\lambda|^{\downarrow|\rho|} \tilde{\chi}_{(\rho, 1|\lambda|-|\rho|)}^{\lambda}, & |\rho| \leqq|\lambda| \\ 0, & |\rho|>|\lambda|\end{cases}
$$

where $n^{\downarrow k}=n(n-1) \cdots(n-k+1)$ is a descending power for $n, k \in \mathbb{N}, k \leqq n$. We use a conventional notation

$$
z_{\rho}=\prod_{i=1}^{\infty} i^{m_{i}(\rho)} m_{i}(\rho) !, \quad \rho=\left(1^{m_{1}(\rho)} 2^{m_{2}(\rho)} \cdots\right) \in \mathbb{Y} .
$$

\section{THEOREM 2.1}

Let $k, n \in \mathbb{N}$, and let $k \leqq n$. For $\Lambda=\left(\lambda^{\zeta}\right)_{\zeta \in \widehat{T}} \in \mathbb{Y}_{k}(\widehat{T})$ and $\mathrm{M}=\left(\mu^{\zeta}\right)_{\zeta \in \widehat{T}} \in \mathbb{Y}_{n}(\widehat{T})$, the Martin kernel is expressed as

$$
K(\Lambda, M)=\frac{n^{k}}{n^{\downarrow k}} \prod_{\zeta \in \widehat{T}}\left\{\frac{1}{(\operatorname{dim} \zeta)^{\mid \lambda^{\zeta \mid}}} \sum_{\rho \in \mathbb{Y}_{|\lambda \zeta|}} \frac{1}{z_{\rho}} \chi_{\rho}^{\lambda^{\zeta}} \frac{1}{n^{\mid \lambda^{\zeta \mid}}} \Sigma_{\rho}\left(\mu^{\zeta}\right)\right\} .
$$

Proof

Note that the right-hand side of (2.3) is actually a finite product since $\lambda^{\zeta}=\varnothing$ holds except for a finite number of $\zeta$ 's. If no paths connect $\Lambda$ to $\mathrm{M}$, there exists $\zeta \in \widehat{T}$ such that $\left|\lambda^{\zeta}\right|>\left|\mu^{\zeta}\right|$. Then, $\Sigma_{\rho}\left(\mu^{\zeta}\right)=0$ holds for any $\rho \in \mathbb{Y}_{|\lambda \zeta|}$ by (2.1), and hence both sides of (2.3) are 0. Assuming that $\left|\lambda^{\zeta}\right| \leqq\left|\mu^{\zeta}\right|$ holds for any $\zeta \in \widehat{T}$, we deduce (2.3) by combining the character formula for $\mathfrak{S}_{k}(T)$ and the Fourier inversion on $\mathfrak{S}_{k}(T)$. Recall (1.28) with (1.25). Then, it suffices to show (2.3) for the case of $T^{\natural}$ :

$$
K^{\natural}(\Lambda, \mathrm{M})=\frac{n^{k}}{n^{\downarrow k}} \prod_{\zeta \in \widehat{T}^{\natural}}\left(\sum_{\rho \in \mathbb{Y}_{|\lambda \zeta|}} \frac{1}{z_{\rho}} \chi_{\rho}^{\lambda^{\zeta}} \frac{1}{n^{|\lambda \zeta|}} \Sigma_{\rho}\left(\mu^{\zeta}\right)\right) .
$$

In this proof below, we write $T, d, k$ instead of $T^{\natural}, d^{\natural}, K^{\natural}$ for simplicity. For the basic element $(\boldsymbol{t}, \sigma)=\boldsymbol{t} \sigma$, where $\sigma=\left(i_{1} i_{2} \cdots i_{l}\right)$, and $\zeta \in \widehat{T}\left(=\widehat{T}^{\natural}\right)$, we simply write

$$
\zeta(\boldsymbol{t})=\zeta\left(t_{i_{l}} \cdots t_{i_{1}}\right) .
$$

Since $T\left(=T^{\natural}\right)$ is commutative, the order of the product of $t_{i_{j}}$ 's is not involved. 
Step 1. Using

$$
\left.\frac{1}{d(\mathrm{M})} \chi^{\mathrm{M}}\right|_{\mathfrak{S}_{k}(T)}=\sum_{\mathrm{N} \in \mathbb{Y}_{k}(\widehat{T})} \frac{d(\mathrm{~N}, \mathrm{M})}{d(\mathrm{M})} \chi^{\mathrm{N}}
$$

and the complete orthonormality of the irreducible characters with respect to the normalized Haar measure $d g$ on $\mathfrak{S}_{k}(T)$, we have that

$$
\begin{aligned}
\frac{d(\Lambda, \mathrm{M})}{d(\mathrm{M})} & =\int_{\mathfrak{S}_{k}(T)} \frac{1}{d(\mathrm{M})} \chi^{\mathrm{M}}(g) \overline{\chi^{\Lambda}(g)} d g \\
& =\sum_{\sigma \in \mathfrak{S}_{k}} \frac{1}{k !} \int_{T^{k}} \frac{1}{d(\mathrm{M})} \chi^{\mathrm{M}}(\boldsymbol{t} \sigma) \overline{\chi^{\Lambda}(\boldsymbol{t} \sigma)} d \boldsymbol{t}
\end{aligned}
$$

where $\boldsymbol{t}=\left(t_{1}, \ldots, t_{k}\right), d \boldsymbol{t}=d t_{1} \cdots d t_{k}$, and $d t$ is the normalized Haar measure on $T$. Let $\sigma \in \mathfrak{S}_{k}$ be fixed (until the end of Step 3) to have cycle decomposition $\sigma=\sigma_{1} \cdots \sigma_{l}$. Decompose $\boldsymbol{t} \in T^{k}$ as $\boldsymbol{t}=\boldsymbol{t}_{0} \boldsymbol{t}_{1} \cdots \boldsymbol{t}_{l}$ so that $\operatorname{supp} \boldsymbol{t}_{j}=\operatorname{supp} \sigma_{j}(j=$ $1, \ldots, l)$ and $\operatorname{supp} \boldsymbol{t}_{0}=\{1, \ldots, k\} \backslash \bigsqcup_{j=1}^{l} \operatorname{supp} \boldsymbol{t}_{j}$. If $\sigma=e_{\mathfrak{S}_{k}}$, we simply set $\boldsymbol{t}_{0}=\boldsymbol{t}$. Letting $\boldsymbol{t}_{0}, \boldsymbol{t}_{1}, \ldots, \boldsymbol{t}_{l}$ vary independently, we compute the integral over $T^{k}$ for each fixed $\sigma \in \mathfrak{S}_{k}$ in (2.4).

Step 2. Applying (1.8) to $\Lambda=\left(\lambda^{\zeta}\right)_{\zeta \in \widehat{T}} \in \mathbb{Y}_{k}(\widehat{T})$ and $g=\boldsymbol{t} \sigma$, we have

$$
\chi^{\Lambda}(\boldsymbol{t} \sigma)=\sum_{\mathcal{Q}, \mathcal{J}} \prod_{\zeta \in \widehat{T}}\left\{\left(\prod_{q \in Q_{\zeta}} \zeta\left(t_{q}\right)\right)\left(\prod_{j \in J_{\zeta}} \zeta\left(\boldsymbol{t}_{j}\right)\right) \chi_{\left(\left|\sigma_{j}\right|\right)_{j \in J_{\zeta}} \sqcup\left(1^{\left|Q_{\zeta}\right|}\right)}^{\lambda^{\zeta}}\right\},
$$

where $(\mathcal{Q}, \mathcal{J})$ satisfies

$$
\left|\lambda^{\zeta}\right|=\left|Q_{\zeta}\right|+\sum_{j \in J_{\zeta}}\left|\sigma_{j}\right|, \quad \zeta \in \widehat{T} .
$$

Note that partitions $\mathcal{Q}$ of $Q$ and $\mathcal{J}$ of $J$ vary independently of $\boldsymbol{t}$ in (2.5). This ensures that integration in $\boldsymbol{t}$ over $T^{k}$ and summation in $\mathcal{Q}, \mathcal{J}$ commute. Applying (1.8) (with the note following the equation) again to $\mathrm{M}=\left(\mu^{\zeta}\right)_{\zeta \in \widehat{T}} \in \mathbb{Y}_{n}(\widehat{T})$, we have

$$
\begin{aligned}
\left.\chi^{\mathrm{M}}\right|_{\mathfrak{S}_{k}(T)}(\boldsymbol{t} \sigma)= & \sum_{\mathcal{Q}, \mathcal{J}} \frac{(n-k) !}{\prod_{\zeta \in \widehat{T}}\left(\left|\mu^{\zeta}\right|-\left|Q_{\zeta}\right|-\sum_{j \in J_{\zeta}}\left|\sigma_{j}\right|\right) !} \\
& \times \prod_{\zeta \in \widehat{T}}\left\{\left(\prod_{q \in Q_{\zeta}} \zeta\left(t_{q}\right)\right)\left(\prod_{j \in J_{\zeta}} \zeta\left(\boldsymbol{t}_{j}\right)\right) \chi_{\left(\left|\sigma_{j}\right|\right)_{j \in J_{\zeta}} \sqcup\left(1^{|\mu \zeta|-\sum_{j \in J_{\zeta}}\left|\sigma_{j}\right|}\right)}^{\mu^{\zeta}}\right\},
\end{aligned}
$$

where $(\mathcal{Q}, \mathcal{J})$ satisfies

$$
\left|\mu^{\zeta}\right| \geqq\left|Q_{\zeta}\right|+\sum_{j \in J_{\zeta}}\left|\sigma_{j}\right|, \quad \zeta \in \widehat{T} .
$$

The $(\mathcal{Q}, \mathcal{J})$ 's in $(2.5)$ and $(2.7)$ are partitions of the same object determined by $\sigma$ with constraints of (2.6) and (2.8), respectively. Since $\left|\lambda^{\zeta}\right| \leqq\left|\mu^{\zeta}\right|$ for any $\zeta \in \widehat{T}$, (2.6) is stronger. Combining (2.5) with (2.7) and taking integration over $T^{k}$, we write 


$$
\begin{aligned}
& \int_{T^{k}} \chi^{\mathrm{M}}(\boldsymbol{t} \sigma) \overline{\chi^{\Lambda}(\boldsymbol{t} \sigma)} d \boldsymbol{t} \\
& =\sum_{\mathcal{Q}, \mathcal{J}} \sum_{\mathcal{Q}^{\prime}, \mathcal{J}^{\prime}} \frac{(n-k) !}{\prod_{\zeta \in \widehat{T}}\left(\left|\mu^{\zeta}\right|-\left|Q_{\zeta}\right|-\sum_{j \in J_{\zeta}}\left|\sigma_{j}\right|\right) !}
\end{aligned}
$$

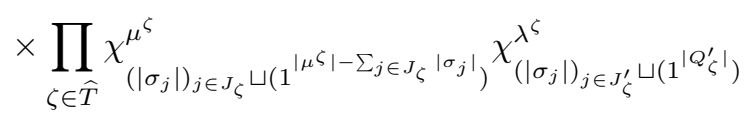

$$
\begin{aligned}
& \times \int_{T^{k}} \prod_{\zeta \in \widehat{T}}\left\{\left(\prod_{q \in Q_{\zeta}} \zeta\left(t_{q}\right)\right)\left(\prod_{q \in Q_{\zeta}^{\prime}} \overline{\zeta\left(t_{q}\right)}\right)\left(\prod_{j \in J_{\zeta}} \zeta\left(\boldsymbol{t}_{j}\right)\right)\left(\prod_{j \in J_{\zeta}^{\prime}} \overline{\zeta\left(\boldsymbol{t}_{j}\right)}\right)\right\} d \boldsymbol{t} .
\end{aligned}
$$

Step 3. We verify that the integral on the right-hand side of (2.9), temporarily labeled by $(*)$ here, is equal to $\delta_{(\mathcal{Q}, \mathcal{J}),\left(\mathcal{Q}^{\prime}, \mathcal{J}^{\prime}\right)}$. Recall that we are considering a partition induced by $\sigma$. Both $\mathcal{Q}$ and $\mathcal{Q}^{\prime}$ give $\widehat{T}$-labeling to the singleton blocks, while $\mathcal{J}$ and $\mathcal{J}^{\prime}$ give $\widehat{T}$-labeling to the other blocks. If $\mathcal{Q} \neq \mathcal{Q}^{\prime}$, then there exists a block $\{q\}$ given distinct labels, say, $\zeta_{1}$ and $\zeta_{2}$, by $\mathcal{Q}$ and $\mathcal{Q}^{\prime}$, respectively. When we compute $(*)$ by using Fubini's theorem, we find

$$
\int_{T} \zeta_{1}\left(t_{q}\right) \overline{\zeta_{2}\left(t_{q}\right)} d t_{q}, \quad \zeta_{1} \neq \zeta_{2}
$$

inside the integral, which vanishes by the orthogonality of (irreducible) characters of $T$. If $\mathcal{J} \neq \mathcal{J}^{\prime}$, then there exists a block $\left\{i_{1}, i_{2}, \ldots, i_{p}\right\}$ with distinct $\widehat{T}$-labeling by $\mathcal{J}$ and $\mathcal{J}^{\prime}$. Letting $\sigma_{j}=\left(i_{1} i_{2} \cdots i_{p}\right)$ and $d \boldsymbol{t}_{j}=d t_{i_{1}} \cdots d t_{i_{p}}$, we have

$$
\int_{T^{p}} \zeta_{1}\left(\boldsymbol{t}_{j}\right) \overline{\zeta_{2}\left(\boldsymbol{t}_{j}\right)} d \boldsymbol{t}_{j}, \quad \zeta_{1} \neq \zeta_{2}
$$

inside the integral, which vanishes also. If $(\mathcal{Q}, \mathcal{J})=\left(\mathcal{Q}^{\prime}, \mathcal{J}^{\prime}\right)$, then we get

$$
(*)=\prod_{\zeta \in \widehat{T}}\left\{\prod_{q \in Q_{\zeta}}\left(\int_{T} \zeta\left(t_{q}\right) \overline{\zeta\left(t_{q}\right)} d t_{q}\right) \prod_{j \in J_{\zeta}}\left(\int_{T^{\left|\sigma_{j}\right|}} \zeta\left(\boldsymbol{t}_{j}\right) \overline{\zeta\left(\boldsymbol{t}_{j}\right)} d \boldsymbol{t}_{j}\right)\right\}=1 .
$$

Moreover, (2.6) with $\left(\mathcal{Q}^{\prime}, \mathcal{J}^{\prime}\right)$ yields

$$
\left|\lambda^{\zeta}\right|=\left|Q_{\zeta}^{\prime}\right|+\sum_{j \in J_{\zeta}^{\prime}}\left|\sigma_{j}\right|=\left|Q_{\zeta}\right|+\sum_{j \in J_{\zeta}}\left|\sigma_{j}\right|
$$

Hence (2.9) now implies that

$$
\begin{aligned}
\int_{T^{k}} \chi^{\mathrm{M}}(\boldsymbol{t} \sigma) \overline{\chi^{\Lambda}(\boldsymbol{t} \sigma)} d \boldsymbol{t}= & \sum_{\mathcal{Q}, \mathcal{J}} \frac{(n-k) !}{\prod_{\zeta \in \widehat{T}}\left(\left|\mu^{\zeta}\right|-\left|\lambda^{\zeta}\right|\right) !} \\
& \times \prod_{\zeta \in \widehat{T}} \chi_{\left(\left|\sigma_{j}\right|\right)_{j \in J_{\zeta}} \sqcup\left(1^{\left|Q_{\zeta}\right|}\right) \sqcup\left(1^{|\mu \zeta|-|\lambda \zeta|)}\right.}^{\chi_{\left(\left|\sigma_{j}\right|\right)_{j \in J_{\zeta}} \sqcup\left(1^{\left|Q_{\zeta}\right|}\right)}^{\lambda^{\zeta}}} .
\end{aligned}
$$

Step 4. Combine (2.4) and (2.10) with

$$
d(\mathrm{M})=\operatorname{dim} \pi^{\mathrm{M}}=\frac{n !}{\prod_{\zeta \in \widehat{T}}\left|\mu^{\zeta}\right| !} \prod_{\zeta \in \widehat{T}} \operatorname{dim} \mu^{\zeta}, \quad \mathrm{M} \in \mathbb{Y}_{n}(\widehat{T}) .
$$


We write $\mathcal{Q}^{\sigma}, \mathcal{J}^{\sigma}$ for partitions in (2.10) since they depend on $\sigma$. Then, we see that

$$
\begin{aligned}
& K(\Lambda, \mathrm{M})=\frac{1}{k ! n^{\downarrow k}} \sum_{\sigma \in \mathfrak{S}_{k}} \sum_{\mathcal{Q}^{\sigma}, \mathcal{J}^{\sigma}} \prod_{\zeta \in \widehat{T}}\left\{\frac{\left|\mu^{\zeta}\right| !}{\left(\left|\mu^{\zeta}\right|-\left|\lambda^{\zeta}\right|\right) !}\right. \\
& \left.\times \frac{1}{\operatorname{dim} \mu^{\zeta}} \chi_{\left(\left|\sigma_{j}\right|\right)_{j \in J} \sigma \sqcup\left(1^{\left|Q_{\zeta}^{\sigma}\right|}\right) \sqcup\left(1^{\prime \mu} \zeta|-| \lambda \zeta \mid\right)}^{\mu^{\zeta}} \chi_{\left(\left|\sigma_{j}\right|\right)_{j \in J_{\zeta}^{\sigma}} \sqcup\left(1^{\left|Q_{\zeta}^{\sigma}\right|}\right)}^{\lambda^{\zeta}}\right\} .
\end{aligned}
$$

Note that $\sum_{\mathcal{Q}^{\sigma}, \mathcal{J}^{\sigma}} \cdots$ in (2.11) actually depends only on the conjugacy class of $\sigma$. We hence continue (2.11) as

$$
\begin{aligned}
K(\Lambda, \mathrm{M})= & \frac{1}{k ! n^{\downarrow k}} \sum_{\rho \in \mathbb{Y}_{k}} \frac{k !}{z_{\rho}} \sum_{\mathcal{Q}^{\sigma}, \mathcal{J}^{\sigma}} \prod_{\zeta \in \widehat{T}}\left\{\frac{\left|\mu^{\zeta}\right| !}{\left(\left|\mu^{\zeta}\right|-\left|\lambda^{\zeta}\right|\right) !}\right. \\
& \left.\times \frac{1}{\operatorname{dim} \mu^{\zeta}} \chi_{\left(\left|\sigma_{j}\right|\right)_{j \in J_{\zeta}} \sqcup\left(1^{\left|Q_{\zeta}^{\sigma}\right|}\right) \sqcup\left(1^{\zeta} \mu^{\zeta}|-| \lambda \zeta \mid\right)} \chi_{\left(\left|\sigma_{j}\right|\right)_{j \in J_{\zeta}^{\sigma}}^{\lambda^{\zeta}}}{ }^{\left.\mid 1^{\left|Q_{\zeta}^{\sigma}\right|}\right)}\right\},
\end{aligned}
$$

where, in the inner sum for each $\rho \in \mathbb{Y}_{k}, \sigma$ is a fixed representative in the conjugacy class $C_{\rho} \subset \mathfrak{S}_{k}$. Recall (2.2) for $z_{\rho}$.

Step 5. Let $\left\{\lambda^{\zeta_{1}}, \ldots, \lambda^{\zeta_{p}}\right\}$ be the set of nontrivial (i.e., not equal to $\varnothing$ ) entries of $\Lambda$. We have $\sum_{i=1}^{p}\left|\lambda^{\zeta_{i}}\right|=k$. Considering the cycles (including trivial ones) of representative $\sigma$ in conjugacy class $C_{\rho}$ labeled by $\zeta_{1}, \ldots, \zeta_{p} \in \widehat{T}$ (where the cycles are all distinguished), we associate to each $\zeta_{i}$ Young diagram

$$
\rho^{(i)} \in \mathbb{Y}_{\left|\lambda^{\zeta_{i}}\right|} \text { such that } \rho^{(1)} \sqcup \cdots \sqcup \rho^{(p)}=\rho .
$$

Namely, labeling which destroys (2.13) is not allowed because of (2.6). Set $\phi$ : $\mathbb{Y}_{\mid \lambda^{\zeta_{1} \mid}} \times \cdots \times \mathbb{Y}_{\left|\lambda^{\zeta_{p}}\right|} \longrightarrow \mathbb{Y}_{k}$ by

$$
\phi\left(\rho^{(1)}, \ldots, \rho^{(p)}\right)=\rho^{(1)} \sqcup \cdots \sqcup \rho^{(p)} .
$$

It suffices to collect $\rho$ 's belonging to the range of $\phi$ in the sum $\sum_{\rho \in \mathbb{Y}_{k}}$ in (2.12). The inner sum $\sum_{\mathcal{Q}^{\sigma}, \mathcal{J}^{\sigma}}$ in (2.12) is divided into partial sums according to the points of fiber $\phi^{-1}(\rho)$, where it is obvious that different points in fiber $\phi^{-1}(\rho)$ never admit a common partition $\left(\mathcal{Q}^{\sigma}, \mathcal{J}^{\sigma}\right)$. Rearranging the right-hand side of (2.12), we have

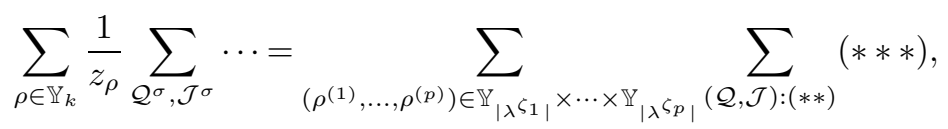

where the range $(* *)$ over which partition $(\mathcal{Q}, \mathcal{J})$ runs depends on $\left(\rho^{(1)}, \ldots, \rho^{(p)}\right)$. On the other hand, $(* * *)$ does not depend on $(\mathcal{Q}, \mathcal{J})$ in $(* *)$ once $\left(\rho^{(1)}, \ldots, \rho^{(p)}\right)$ is chosen, which is equal to

$$
\begin{array}{r}
\frac{1}{\prod_{j=1}^{\infty} j^{m_{j}\left(\rho^{(1)}\right)+\cdots+m_{j}\left(\rho^{(p)}\right)}\left(m_{j}\left(\rho^{(1)}\right)+\cdots+m_{j}\left(\rho^{(p)}\right)\right) !} \\
\cdot \prod_{i=1}^{p}\left\{\frac{\left|\mu^{\zeta_{i}}\right| !}{\left(\left|\mu^{\zeta_{i}}\right|-\left|\lambda^{\zeta_{i}}\right|\right) !} \frac{1}{\operatorname{dim} \mu \zeta_{i}} \chi_{\rho^{(i)} \sqcup\left(1\left|\mu \zeta^{\zeta_{i}|-| \lambda} \zeta_{i}\right|\right.}^{\mu^{\zeta_{i}}} \chi_{\rho^{(i)}}^{\lambda^{\zeta_{i}}}\right\} .
\end{array}
$$


The cardinality of the range $(* *)$ for a given $\left(\rho^{(1)}, \ldots, \rho^{(p)}\right)$ is

$$
\prod_{j=1}^{\infty} \frac{\left(m_{j}\left(\rho^{(1)}\right)+\cdots+m_{j}\left(\rho^{(p)}\right)\right) !}{m_{j}\left(\rho^{(1)}\right) ! \cdots m_{j}\left(\rho^{(p)}\right) !}
$$

since the cycles of length $j$ admit labeling by $\zeta_{1}, \ldots, \zeta_{p}$ in the $j$ th factor ways in (2.16).

Step 6. Combining (2.12) with (2.14)-(2.16), we have

$$
\begin{aligned}
& K(\Lambda, \mathrm{M})=\frac{1}{n^{\downarrow k}} \sum_{\left(\rho^{(1)}, \ldots, \rho^{(p)}\right) \in \mathbb{Y}_{\left|\lambda \zeta_{1}\right|} \times \cdots \times \mathbb{Y}_{\left|\lambda^{\lambda} \zeta_{p}\right|}} \frac{1}{z_{\rho^{(1)}} \cdots z_{\rho^{(p)}}} \\
& \times \prod_{i=1}^{p}\left\{\frac{\left|\mu^{\zeta_{i}}\right| !}{\left(\left|\mu^{\zeta_{i}}\right|-\left|\lambda \zeta_{i}\right|\right) !} \frac{1}{\operatorname{dim} \mu^{\zeta_{i}}} \chi_{\rho^{(i)} \sqcup(1 \mid \mu}^{\mu^{\zeta_{i}}}\right.
\end{aligned}
$$

then, by using the notation of (2.1) and noting that $k=\sum_{i=1}^{p}\left|\lambda^{\zeta_{i}}\right|$, we continue (2.17) as

$$
\begin{aligned}
& =\frac{n^{k}}{n^{\downarrow k}} \sum_{\left(\rho^{(1)}, \ldots, \rho^{(p)}\right) \in \mathbb{Y}_{\left|\lambda \zeta_{1}\right|} \times \cdots \times \mathbb{Y}_{\mid \lambda} \zeta_{p} \mid} \frac{1}{z_{\rho^{(1)}} \cdots z_{\rho^{(p)}}} \prod_{i=1}^{p}\left\{\frac{\Sigma_{\rho^{(i)}}\left(\mu^{\zeta_{i}}\right)}{n^{\left|\lambda^{\zeta_{i}}\right|}} \chi_{\rho^{(i)}}^{\lambda^{\zeta_{i}}}\right\} \\
& =\frac{n^{k}}{n^{\downarrow k}} \prod_{i=1}^{p}\left(\sum_{\rho \in \mathbb{Y}_{\mid \lambda} \zeta_{i} \mid} \frac{1}{z_{\rho}} \frac{\Sigma_{\rho}\left(\mu^{\zeta_{i}}\right)}{n^{\left|\lambda^{\zeta_{i}}\right|}} \chi_{\rho}^{\lambda^{\zeta_{i}}}\right)=\frac{n^{k}}{n^{\downarrow k}} \prod_{\zeta \in \widehat{T}}\left(\sum_{\rho \in \mathbb{Y}_{\mid \lambda} \zeta_{\mid}} \frac{1}{z_{\rho}} \chi_{\rho}^{\lambda^{\zeta}} \frac{\Sigma_{\rho}\left(\mu^{\zeta}\right)}{n^{\left|\lambda^{\zeta}\right|}}\right) .
\end{aligned}
$$

This completes the proof of (2.3).

We investigate the asymptotic behavior of (2.3) with $k=|\Lambda|=\sum_{\zeta \in \widehat{T}}\left|\lambda^{\zeta}\right|$ fixed as $n=|\mathrm{M}|=\sum_{\zeta \in \widehat{T}}\left|\mu^{\zeta}\right|$ tends to $\infty$. A suitable framework for the analysis of the function $n^{-\left|\lambda^{\zeta}\right|} \Sigma_{\rho}\left(\mu^{\zeta}\right)$ is given by the Kerov-Olshanski algebra $\mathbb{A}$ consisting of polynomial functions of Young diagrams, which we briefly review now.

We set the Frobenius coordinates of $\mu=\left(\mu_{1} \geqq \mu_{2} \geqq \cdots\right) \in \mathbb{Y}$ by

$$
a_{i}(\mu)=\mu_{i}-i+\frac{1}{2}, \quad b_{i}(\mu)=\mu_{i}^{\prime}-i+\frac{1}{2}, \quad i=1, \ldots, d(\mu),
$$

where $d=d(\mu)$ is the diagonal length (number of boxes) of $\mu$, and write them as $\mu=\left(a_{i}(\mu) \mid b_{i}(\mu)\right)_{i=1, \ldots, d}$. For $k \in \mathbb{N}$, the (supersymmetric) power sum in $\mu$ is defined by

$$
p_{k}=p_{k}(\mu)=\sum_{i=1}^{d} a_{i}(\mu)^{k}+(-1)^{k-1} b_{i}(\mu)^{k}, \quad \mu \in \mathbb{Y},
$$

and by

$$
p_{\rho}=p_{\rho}(\mu)=p_{\rho_{1}}(\mu) p_{\rho_{2}}(\mu) \cdots p_{\rho_{l}}(\mu), \quad \mu \in \mathbb{Y},
$$

for arbitrary $\rho \in \mathbb{Y}$ with $l=l(\rho)$. Power sums $\left\{p_{k}\right\}_{k \in \mathbb{N}}$, regarded as functions in $\mu$, are algebraically independent over $\mathbb{R}$. The algebra generated by $\left\{p_{k}\right\}_{k \in \mathbb{N}}$ is called the Kerov-Olshanski algebra and is denoted by $\mathbb{A}$. Declaring $p_{k}$ to be homogeneous of degree $k$, written as $\operatorname{deg} p_{k}=k$, we equip $\mathbb{A}$ with the canonical 
filtration. The canonical degree of any element $f \in \mathbb{A}$ is also denoted by $\operatorname{deg} f$. For example, $\operatorname{deg} p_{\rho}=\rho_{1}+\cdots+\rho_{l}=|\rho|$ for $\rho \in \mathbb{Y}$. We have that $\Sigma_{\rho}$ in (2.1) also belongs to $\mathbb{A}$. It is related to a power sum as follows.

\section{PROPOSITION 2.2}

For any $\rho \in \mathbb{Y}$, there exists $R_{\rho} \in \mathbb{A}$ such that

$$
\Sigma_{\rho}=p_{\rho}+R_{\rho}, \quad \operatorname{deg} R_{\rho}<|\rho|
$$

hold in $\mathbb{A}$.

We refer to [10, Sections 3 and 4] for the proof of Proposition 2.2.

The expressions from (2.21) combined with (2.19) and (2.20) suggest that the convergence of $K(\Lambda, \mathrm{M})$ in (2.3) as $n=|\mathrm{M}| \rightarrow \infty$ is characterized by the asymptotics of rescaled Frobenius coordinates

$$
\frac{a_{i}\left(\mu^{\zeta}\right)}{n}, \quad \frac{b_{i}\left(\mu^{\zeta}\right)}{n}, \quad \zeta \in \widehat{T}, i \in \mathbb{N} .
$$

We are thus in a position to get a concrete expression of the Martin boundary and the Martin kernels (1.33) of the branching graph $\mathbb{Y}(\widehat{T})$. Set

$$
\begin{aligned}
\widetilde{\Delta}= & \left\{(\alpha, \beta, c) \mid \alpha=\left(\alpha_{\zeta, i}\right)_{\zeta \in \widehat{T}, i \in \mathbb{N}}, \beta=\left(\beta_{\zeta, i}\right)_{\zeta \in \widehat{T}, i \in \mathbb{N}}, c=\left(c_{\zeta}\right)_{\zeta \in \widehat{T}} ;\right. \\
& \alpha_{\zeta, 1} \geqq \alpha_{\zeta, 2} \geqq \cdots \geqq 0, \beta_{\zeta, 1} \geqq \beta_{\zeta, 2} \geqq \cdots \geqq 0, c_{\zeta} \geqq 0, \\
& \left.\sum_{i=1}^{\infty}\left(\alpha_{\zeta, i}+\beta_{\zeta, i}\right) \leqq c_{\zeta} \text { for } \forall \zeta \in \widehat{T} ; \sum_{\zeta \in \widehat{T}} c_{\zeta} \leqq 1\right\}
\end{aligned}
$$

and

$$
\Delta=\left\{(\alpha, \beta, c) \in \widetilde{\Delta} \mid \sum_{\zeta \in \widehat{T}} c_{\zeta}=1\right\} .
$$

We have

$$
\Delta \varsubsetneqq \widetilde{\Delta} \subset B\left(\ell^{1}(\widehat{T} \times \mathbb{N})\right)^{2} \times B\left(\ell^{1}(\widehat{T})\right),
$$

where $B(\cdot)$ denotes the closed unit ball with center 0 in an $\ell^{1}$-space. The closed unit balls in the rightmost set of (2.24) are compact with respect to the weak* topologies $\sigma\left(\ell^{1}, c_{0}\right)$, where $c_{0}$ consists of the sequences converging to 0 at infinity. In this case, the product of the weak* topologies is equivalent to the topology of pointwise converging on $(\widehat{T} \times \mathbb{N}) \sqcup(\widehat{T} \times \mathbb{N}) \sqcup \widehat{T}$. Since $\widetilde{\Delta}$ is closed with respect to this pointwise converging topology as directly seen from the definition $(2.22), \widetilde{\Delta}$ is a compact set. On the other hand, we should note that $\Delta$ of (2.23) is not closed in $\widetilde{\Delta}$ with respect to the given topology if $\widehat{T}$ is an infinite set. As (supersymmetric) power sums and Schur functions on $\widetilde{\Delta}$, let us set

$$
\begin{aligned}
& p_{k}^{\zeta}(\alpha, \beta, c)= \begin{cases}\sum_{i=1}^{\infty}\left(\alpha_{\zeta, i}^{k}+(-1)^{k-1} \beta_{\zeta, i}^{k}\right), & k \geqq 2, k \in \mathbb{N}, \\
c_{\zeta}, & k=1,\end{cases} \\
& p_{\rho}^{\zeta}(\alpha, \beta, c)=p_{\rho_{1}}^{\zeta}(\alpha, \beta, c) \cdots p_{\rho_{l}}^{\zeta}(\alpha, \beta, c), \quad \rho=\left(\rho_{1} \geqq \cdots \geqq \rho_{l}\right) \in \mathbb{Y},
\end{aligned}
$$


with $p_{\varnothing}^{\zeta}(\alpha, \beta, c)=1$, and

$$
s_{\lambda}^{\zeta}(\alpha, \beta, c)=\sum_{\rho \in \mathbb{Y}_{|\lambda|}} \frac{1}{z_{\rho}} \chi_{\rho}^{\lambda} p_{\rho}^{\zeta}(\alpha, \beta, c), \quad \lambda \in \mathbb{Y},
$$

for $\zeta \in \widehat{T}$ and $(\alpha, \beta, c) \in \widetilde{\Delta}$.

The definition of (2.27) obviously comes from the well-known relation (or Frobenius character formula) between Schur functions and power sums. Note that

$$
p_{1}^{\zeta}(\alpha, \beta, c) \neq \sum_{i=1}^{\infty}\left(\alpha_{\zeta, i}+\beta_{\zeta, i}\right)
$$

in general.

\section{LEMMA 2.3}

(a) Let $T$ be finite.

(1) $p_{k}^{\zeta} \in C(\Delta ; \mathbb{R}(=\{\mathbb{R}$-valued continuous functions on $\Delta\}))$. Hence $p_{\rho}^{\zeta}, s_{\lambda}^{\zeta} \in$ $C(\Delta ; \mathbb{R})$ also.

(2) $\left\{p_{k}^{\zeta} \mid \zeta \in \widehat{T}, k \in \mathbb{N}\right\}$ separates two arbitrary points in $\Delta$.

(b) Let $T$ be infinite. Then, (1) and (2) above hold with $\widetilde{\Delta}$ replacing $\Delta$.

\section{Proof}

(a.1) The continuity of $p_{k}^{\zeta}$ with respect to the pointwise converging topology on $\Delta$ is easy to see, which is similar to the well-known argument on the Thoma simplex (i.e., $\Delta$ for $T=\{e\}$ ). (See also [9, Lemma 4.6].)

(a.2) This separation property is also similar to the case of the Thoma simplex. Let $(\alpha, \beta, c),\left(\alpha^{\prime}, \beta^{\prime}, c^{\prime}\right) \in \Delta$ satisfy

$$
p_{k}^{\zeta}(\alpha, \beta, c)=p_{k}^{\zeta}\left(\alpha^{\prime}, \beta^{\prime}, c^{\prime}\right), \quad \zeta \in \widehat{T}, k \in \mathbb{N} .
$$

Setting $k=1$, we have that $\left(c_{\zeta}\right)_{\zeta \in \widehat{T}}=\left(c_{\zeta}^{\prime}\right)_{\zeta \in \widehat{T}}$. For each $\zeta \in \widehat{T},\left\{p_{k}^{\zeta}(\alpha, \beta, c) \mid k \in\right.$ $\mathbb{N}\}$ completely determines $\left(\alpha_{\zeta, i}\right)_{i \in \mathbb{N}}$ and $\left(\beta_{\zeta, i}\right)_{i \in \mathbb{N}}$ through the equality

$$
\begin{aligned}
\exp \left\{\sum_{k=2}^{\infty} \frac{p_{k}^{\zeta}(\alpha, \beta, c)}{k} z^{k}\right\} & =\exp \left\{\sum_{k=2}^{\infty} \sum_{i=1}^{\infty}\left(\alpha_{\zeta, i}^{k}+(-1)^{k-1} \beta_{\zeta, i}^{k}\right) \frac{z^{k}}{k}\right\} \\
& =\exp \left\{-\sum_{i=1}^{\infty}\left(\alpha_{\zeta, i}+\beta_{\zeta, i}\right) z\right\} \prod_{i=1}^{\infty} \frac{1+\beta_{\zeta, i} z}{1-\alpha_{\zeta, i} z}
\end{aligned}
$$

The proof in the case of (b) is quite similar.

\section{LEMMA 2.4}

Let $X$ be a compact set, and let $\left\{h_{\alpha}\right\}_{\alpha \in A} \subset C(X ; \mathbb{R})$ separate two arbitrary points in $X$. Then, the topology on $X$ determined by pointwise converging on $A$ through $x(\alpha)=h_{\alpha}(x)$ coincides with the original one on $X$. 
Proof

The assertion is immediate from the compactness of $X$, the continuity of $h_{\alpha}$, and the fact that the pointwise converging topology is Hausdorff.

\section{THEOREM 2.5}

A homeomorphic characterization of the Martin boundary $\partial \mathbb{Y}(\widehat{T})$ of the branching graph $\mathbb{Y}(\widehat{T})$ is given by the following:

(a) if $T$ is finite, then $\partial \mathbb{Y}(\widehat{T}) \cong \Delta$;

(b) if $T$ is infinite, then $\partial \mathbb{Y}(\widehat{T}) \cong \widetilde{\Delta}$.

The Martin kernel is expressed by using Schur functions (2.27) as

$$
K(\Lambda, \omega)=\prod_{\zeta \in \widehat{T}} \frac{1}{(\operatorname{dim} \zeta)^{|\lambda \zeta|}} s_{\lambda \zeta}^{\zeta}(\omega), \quad \Lambda=\left(\lambda^{\zeta}\right)_{\zeta \in \widehat{T}} \in \mathbb{Y}(\widehat{T}), \omega=(\alpha, \beta, c),
$$

where $\omega \in \Delta$ for finite $T$ and $\omega \in \widetilde{\Delta}$ for infinite $T$.

Proof

The right-hand side of (2.28) is actually a finite product by the definitions (2.25)(2.27).

Step 1. A point in the Martin boundary $\partial \mathbb{Y}(\widehat{T})$ is given by an equivalence class of Cauchy sequences $\left\{\mathrm{M}^{(n)}\right\}_{n \in \mathbb{N}}$ satisfying (1.31) and (1.32). With $k \in \mathbb{N}$ and $\Lambda \in \mathbb{Y}_{k}(\widehat{T})$ being fixed, let $K\left(\Lambda, \mathrm{M}^{(n)}\right)$ converge with $m=\left|\mathrm{M}^{(n)}\right| \rightarrow \infty$ as $n \rightarrow \infty$, where $\mathrm{M}^{(n)}=\left(\mu^{(n) \zeta}\right)_{\zeta \in \widehat{T}} \in \mathbb{Y}_{m}(\widehat{T})$. In order to simplify the notation, however, we omit the superscript ${ }^{(n)}$ of $\mathrm{M}^{(n)}$ and $\mu^{(n) \zeta}$, and consider the limit with $m=|\mathrm{M}|=\sum_{\zeta \in \widehat{T}}\left|\mu^{\zeta}\right| \rightarrow \infty$. Theorem 2.1 yields that

$$
\prod_{\zeta \in \widehat{T}}\left\{\sum_{\rho \in \mathbb{Y}_{|\lambda \zeta|}} \frac{1}{z_{\rho}} \chi_{\rho}^{\lambda^{\zeta}} \frac{1}{m^{\left|\lambda^{\zeta}\right|}} \Sigma_{\rho}\left(\mu^{\zeta}\right)\right\}
$$

converges with $m \rightarrow \infty$. We verify that the expression obtained by replacing $\Sigma_{\rho}$ by $p_{\rho}$ in (2.29) also converges as $m \rightarrow \infty$. Take $R_{\rho} \in \mathbb{A}$ satisfying (2.21) for each $\rho \in \mathbb{Y}$, and consider

$$
\begin{aligned}
& \mid \prod_{\zeta \in \widehat{T}}\left\{\sum_{\rho \in \mathbb{Y}_{|\lambda \zeta|}} \frac{1}{z_{\rho}} \chi_{\rho}^{\lambda^{\zeta}} \frac{1}{m^{\left|\lambda^{\zeta}\right|}}\left(p_{\rho}\left(\mu^{\zeta}\right)+R_{\rho}\left(\mu^{\zeta}\right)\right)\right\} \\
& -\prod_{\zeta \in \widehat{T}}\left\{\sum_{\rho \in \mathbb{Y}_{|\lambda \zeta|}} \frac{1}{z_{\rho}} \chi_{\rho}^{\lambda^{\zeta}} \frac{1}{m^{\left|\lambda^{\zeta}\right|}} p_{\rho}\left(\mu^{\zeta}\right)\right\} \mid .
\end{aligned}
$$

We have that $n^{-\left|\lambda^{\zeta}\right|}\left|p_{\rho}\left(\mu^{\zeta}\right)\right| \leqq 1$ for $\rho \in \mathbb{Y}_{|\lambda \zeta|}$. For a fixed $k \in \mathbb{N}$, the range of $\left|\lambda^{\zeta}\right|$ and $\rho \in \mathbb{Y}_{\left|\lambda^{\zeta}\right|}$ is a finite set because of $k=|\Lambda|=\sum_{\zeta \in \widehat{T}}\left|\lambda^{\zeta}\right|$. Additionally, $R_{\rho}$ is a linear combination of $p_{\sigma}$ 's with $|\sigma|<|\rho|=\left|\lambda^{\zeta}\right|$. Hence there exists $c_{k}>0$, independent of $\zeta \in \widehat{T}$, such that

$$
\left|\sum_{\rho \in \mathbb{Y}_{|\lambda \zeta|}} \frac{1}{z_{\rho}} \chi_{\rho}^{\lambda^{\zeta}} \frac{1}{m^{\left|\lambda^{\zeta}\right|}} p_{\rho}\left(\mu^{\zeta}\right)\right| \leqq \operatorname{dim} \lambda^{\zeta} \sum_{\rho \in \mathbb{Y}_{|\lambda \zeta|}} \frac{1}{z_{\rho}} \leqq c_{k}
$$




$$
\left|\sum_{\rho \in \mathbb{Y}_{|\lambda \zeta|}} \frac{1}{z_{\rho}} \chi_{\rho}^{\lambda^{\zeta}} \frac{1}{m^{\left|\lambda^{\zeta}\right|}} R_{\rho}\left(\mu^{\zeta}\right)\right| \leqq \frac{\operatorname{dim} \lambda^{\zeta}}{m} \sum_{\rho \in \mathbb{Y}_{|\lambda \zeta|}} \frac{1}{z_{\rho} m^{\left|\lambda^{\zeta}\right|-1}}\left|R_{\rho}\left(\mu^{\zeta}\right)\right| \leqq \frac{c_{k}}{m} .
$$

Taking a constant $C_{k}>0$ depending only on $k$, we have that

$$
(2.30) \leqq \frac{1}{m} C_{k}
$$

This together with (2.29) implies that

$$
\prod_{\zeta \in \widehat{T}}\left\{\sum_{\rho \in \mathbb{Y}_{|\lambda \zeta|}} \frac{1}{z_{\rho}} \chi_{\rho}^{\lambda^{\zeta}} \frac{1}{m^{|\lambda \zeta|}} p_{\rho}\left(\mu^{\zeta}\right)\right\}
$$

converges with $m=|\mathrm{M}| \rightarrow \infty$.

Step 2. Since (2.32) converges with $m \rightarrow \infty$ for any $k \in \mathbb{N}$ and any $\Lambda=$ $\left(\lambda^{\zeta}\right)_{\zeta \in \widehat{T}} \in \mathbb{Y}_{k}(\widehat{T})$, we have, equivalently, the convergence of

$$
\begin{aligned}
\frac{1}{m} p_{1}\left(\mu^{\zeta}\right) & =\frac{\left|\mu^{\zeta}\right|}{m} \\
\frac{1}{m^{k}} p_{k}\left(\mu^{\zeta}\right) & =\sum_{i=1}^{\infty}\left\{\left(\frac{a_{i}\left(\mu^{\zeta}\right)}{m}\right)^{k}+(-1)^{k-1}\left(\frac{b_{i}\left(\mu^{\zeta}\right)}{m}\right)^{k}\right\}, \quad k \geqq 2,
\end{aligned}
$$

with $m=|\mathrm{M}| \rightarrow \infty$. Or, in terms of $p_{k}^{\zeta}$ in $(2.25)$,

$$
p_{k}^{\zeta}\left(\left(\frac{a_{i}\left(\mu^{\eta}\right)}{m}\right)_{\eta, i},\left(\frac{b_{i}\left(\mu^{\eta}\right)}{m}\right)_{\eta, i},\left(\frac{\left|\mu^{\eta}\right|}{m}\right)_{\eta}\right)
$$

converges for any $\zeta \in \widehat{T}$ and any $k \in \mathbb{N}$. Lemmas 2.3 and 2.4 then ensure that

$$
\left(\left(\frac{a_{i}\left(\mu^{\zeta}\right)}{m}\right)_{\zeta, i},\left(\frac{b_{i}\left(\mu^{\zeta}\right)}{m}\right)_{\zeta, i},\left(\frac{\left|\mu^{\zeta}\right|}{m}\right)_{\zeta}\right)
$$

converges in $\widetilde{\Delta}$ (resp., $\Delta$ ) for infinite (resp., finite) $T$ with $m=|\mathrm{M}| \rightarrow \infty$. Set its limit as $\left(\left(\alpha_{\zeta, i}\right),\left(\beta_{\zeta, i}\right),\left(c_{\zeta}\right)\right) \in \widetilde{\Delta}$ (resp., $\left.\Delta\right)$. We thus obtain a map from $\partial \mathbb{Y}(\widehat{T})$ into $\widetilde{\Delta}$ (resp., $\Delta$ ) which is homeomorphic again by Lemmas 2.3 and 2.4 .

Step 3. We show that the map $\partial \mathbb{Y}(\widehat{T}) \longrightarrow \widetilde{\Delta}$ (resp., $\Delta$ ) defined in Step 2 is surjective. By virtue of Lemmas 2.3 and 2.4, it suffices to verify that, given $(\alpha, \beta, c) \in \widetilde{\Delta}$ (resp., $\Delta)$ where $\alpha=\left(\alpha_{\zeta, i}\right), \beta=\left(\beta_{\zeta, i}\right)$, and $c=\left(c_{\zeta}\right)$, there exists a sequence $\left(\mathrm{M}=\left(\mu^{\zeta}\right)_{\zeta \in \widehat{T}}\right), \mathrm{M} \in \mathbb{Y}_{n}(\widehat{T})$, satisfying

$$
\lim _{n \rightarrow \infty}\left(\left(\frac{a_{i}\left(\mu^{\zeta}\right)}{n}\right)_{\zeta, i},\left(\frac{b_{i}\left(\mu^{\zeta}\right)}{n}\right)_{\zeta, i},\left(\frac{\left|\mu^{\zeta}\right|}{n}\right)_{\zeta}\right)=(\alpha, \beta, c) .
$$

For simplicity of expressions, we omit precise estimates of the effects caused by taking integer parts. First, according to $c_{\zeta}$, assign $n c_{\zeta}$ (though it is $\left\lfloor n c_{\zeta}\right\rfloor$ with precision) boxes to each $\zeta \in \widehat{T}$ to construct Young diagram $\mu^{\zeta}$. If $T$ is finite, then it is easy to handle the noninteger part $n c_{\zeta}-\left\lfloor n c_{\zeta}\right\rfloor$. If $T$ is infinite, there may remain boxes of order $n$ because of $\sum_{\zeta \in \widehat{T}} c_{\zeta} \leqq 1$. To deal with the remaining boxes, assign $\sqrt{n}$ order number of boxes to $\sqrt{n}$ order number of $\zeta$ 's. Then, we have $\left|\mu^{\zeta}\right| / n \rightarrow c_{\zeta}$ as $n \rightarrow \infty$. For determining $a_{i}\left(\mu^{\zeta}\right)$ and $b_{i}\left(\mu^{\zeta}\right)$ to construct 
$\mu^{\zeta}$, we assign $n \alpha_{\zeta, i}$ and $n \beta_{\zeta, i}$, respectively, to $(\zeta, i) \in \widehat{T} \times \mathbb{N}$ by using $\left\lfloor n c_{\zeta}\right\rfloor$ boxes assigned to $\zeta \in \widehat{T}$ above. This can be done since $\sum_{i=1}^{\infty}\left(\alpha_{\zeta, i}+\beta_{\zeta, i}\right) \leqq c_{\zeta}$ holds. The possible number of remaining boxes at $\zeta \in \widehat{T}$ is at most of order $n$. Again, we consume the remainders by assigning $\sqrt{n}$ order boxes to $\sqrt{n}$ order $i$ 's. The construction immediately yields the convergence of (2.34) in $\widetilde{\Delta}$ (resp., $\Delta$ ). This completes the proof of $\partial \mathbb{Y}(\widehat{T}) \cong \Delta$ for finite $T$ and $\partial \mathbb{Y}(\widehat{T}) \cong \widetilde{\Delta}$ for infinite $T$.

Step 4. We deduce (2.28) from (2.3). Since we established a homeomorphic characterization of $\partial \mathbb{Y}(\widehat{T})$, it suffices to show that, for $\Lambda=\left(\lambda^{\zeta}\right)_{\zeta \in \widehat{T}} \in \mathbb{Y}_{k}(\widehat{T})$ and $\omega=(\alpha, \beta, c)(\in \Delta$ for finite $T$ and $\in \widetilde{\Delta}$ for infinite $T)$, (2.32) converges to $\prod_{\zeta \in \widehat{T}} s_{\lambda \zeta}^{\zeta}(\alpha, \beta, c)$ under the convergence of (2.34) as $m=|\mathrm{M}|=\sum_{\zeta \in \widehat{T}}\left|\mu^{\zeta}\right| \rightarrow \infty$. Recall that

$$
\begin{aligned}
\frac{1}{m} p_{1}\left(\mu^{\zeta}\right) & =\frac{\left|\mu^{\zeta}\right|}{m} \\
\frac{1}{m^{k}} p_{k}\left(\mu^{\zeta}\right) & =\sum_{i=1}^{\infty}\left\{\left(\frac{a_{i}\left(\mu^{\zeta}\right)}{m}\right)^{k}+(-1)^{k-1}\left(\frac{b_{i}\left(\mu^{\zeta}\right)}{m}\right)^{k}\right\}, \quad k \geqq 2 .
\end{aligned}
$$

As in the argument in Lemma 2.3(a), (2.34) yields

$$
\frac{1}{m^{k}} p_{k}\left(\mu^{\zeta}\right) \longrightarrow p_{k}^{\zeta}(\alpha, \beta, c), \quad k \in \mathbb{N}, \zeta \in \widehat{T}
$$

Since the $\zeta$ 's with $\left|\lambda^{\zeta}\right|>0$ are finite, the desired convergence then follows from the definition of $s_{\lambda}^{\zeta}$ in $(2.27)$.

\section{REMARK 2.6}

If $T$ is a trivial group $\left\{e_{T}\right\}$, then $\Delta$ of (2.23) reduces to the Thoma simplex, which is homeomorphic to the Martin boundary of the usual Young graph. Theorem 2.5 under the restriction of $T=\{e\}$ is of course consistent with the well-known result for the Young graph.

As a standard fact, $K(\cdot, \omega)$ satisfies the following superharmonicity property.

LEMMA 2.7

The Martin kernel $K(\Lambda, \omega)$ is superharmonic in $\Lambda$ :

$$
K(\Lambda, \omega) \geqq \sum_{\mathrm{N}: \Lambda \nearrow \mathrm{N}} \kappa(\Lambda, \mathrm{N}) K(\mathrm{~N}, \omega), \quad \Lambda \in \mathbb{Y}(\widehat{T}), \omega \in \partial \mathbb{Y}(\widehat{T}) .
$$

Proof

We have that

$$
K(\Lambda, \omega)=\lim _{m \rightarrow \infty} K(\Lambda, M), \quad \Lambda \in \mathbb{Y}(\widehat{T}),
$$

for an appropriate sequence such that $\mathrm{M} \rightarrow \omega$ with $m=|\mathrm{M}| \rightarrow \infty$. Then, 


$$
\begin{aligned}
\sum_{\mathrm{N}: \Lambda \nearrow \mathrm{N}} \kappa(\Lambda, \mathrm{N}) K(\mathrm{~N}, \omega) & =\sum_{\mathrm{N}: \Lambda \nearrow \mathrm{N}} \kappa(\Lambda, \mathrm{N}) \lim _{m \rightarrow \infty} K(\mathrm{~N}, \mathrm{M}) \\
& \leqq \liminf _{m \rightarrow \infty} \sum_{\mathrm{N}: \Lambda \nearrow \mathrm{N}} \kappa(\Lambda, \mathrm{N}) K(\mathrm{~N}, \mathrm{M})=\liminf _{m \rightarrow \infty} K(\Lambda, \mathrm{M}) \\
& =K(\Lambda, \omega) .
\end{aligned}
$$

\section{LEMMA 2.8}

The Martin kernel $K(\cdot, \omega)$ is harmonic if and only if $\omega \in \Delta$.

Proof

Definitions (2.25)-(2.27) imply that

$$
s_{\varnothing}^{\zeta}(\omega)=p_{\varnothing}^{\zeta}(\omega)=1, \quad s_{(1)}^{\zeta}(\omega)=p_{1}^{\zeta}(\omega)=c_{\zeta}, \quad \omega=(\alpha, \beta, c), c=\left(c_{\zeta}\right)_{\zeta \in \widehat{T}},
$$

for $\zeta \in \widehat{T}$. Moreover, in a similar way to Pieri's formula for Schur functions, we have

$$
s_{(1)}^{\zeta}(\omega) s_{\lambda}^{\zeta}(\omega)=\sum_{\mu \in \mathbb{Y}: \lambda \nearrow \mu} s_{\mu}^{\zeta}(\omega), \quad \lambda \in \mathbb{Y}
$$

for $\zeta \in \widehat{T}$. First, setting $\Lambda=\varnothing$ in (2.28), we have that $K(\varnothing, \omega)=1$ and

$$
\sum_{\mathrm{N} \in \mathbb{Y}(\widehat{T}): \varnothing \nearrow \mathrm{N}} \kappa(\varnothing, \mathrm{N}) K(\mathrm{~N}, \omega)=\sum_{\zeta \in \widehat{T}} s_{(1)}^{\zeta}(\omega)=\sum_{\zeta \in \widehat{T}} c_{\zeta} .
$$

Hence, the harmonicity of $K(\cdot, \omega)$ yields $\omega \in \Delta$ in particular. More generally, setting $\operatorname{supp} \Lambda=\left\{\zeta \in \widehat{T} \mid \lambda^{\zeta} \neq \varnothing\right\}$ for $\Lambda=\left(\lambda^{\zeta}\right)_{\zeta \in \widehat{T}} \in \mathbb{Y}(\widehat{T})$, we see from (2.28) and (2.36) that

$$
\begin{aligned}
\sum_{\mathrm{N}: \Lambda \nearrow \mathrm{N}} & \kappa(\Lambda, \mathrm{N}) K(\mathrm{~N}, \omega) \\
= & \sum_{\zeta \notin \operatorname{supp} \Lambda} \operatorname{dim} \zeta \frac{s_{(1)}^{\zeta}(\omega)}{\operatorname{dim} \zeta} K(\Lambda, \omega) \\
& +\sum_{\zeta \in \operatorname{supp} \Lambda} \operatorname{dim} \zeta\left(\prod_{\eta \in \operatorname{supp} \Lambda \backslash\{\zeta\}} \frac{s_{\lambda^{\eta}}^{\eta}(\omega)}{(\operatorname{dim} \eta)^{\left|\lambda^{\eta}\right|}}\right)\left(\sum_{\mu: \lambda^{\zeta} \nearrow_{\mu}} \frac{s_{\mu}^{\zeta}(\omega)}{(\operatorname{dim} \zeta)^{|\mu|}}\right) \\
= & \sum_{\zeta \notin \operatorname{supp} \Lambda} c_{\zeta} K(\Lambda, \omega) \\
& +\sum_{\zeta \in \operatorname{supp} \Lambda} \frac{1}{(\operatorname{dim} \zeta)^{\left|\lambda^{\zeta}\right|}}\left(\prod_{\eta \in \operatorname{supp} \Lambda \backslash\{\zeta\}} \frac{s_{\lambda^{\eta}}^{\eta}(\omega)}{(\operatorname{dim} \eta)^{\left|\lambda^{\eta}\right|}}\right) \sum_{\mu: \lambda \zeta \nearrow \mu} s_{\mu}^{\zeta}(\omega) \\
= & \sum_{\zeta \notin \operatorname{supp} \Lambda} c_{\zeta} K(\Lambda, \omega)+\sum_{\zeta \in \operatorname{supp} \Lambda} c_{\zeta} K(\Lambda, \omega)=K(\Lambda, \omega)\left(\sum_{\zeta \in \widehat{T}} c_{\zeta}\right) .
\end{aligned}
$$

This yields the assertion. 
The above characterization of the harmonicity of $K(\cdot, \omega)$ is used in the proof of Theorem 3.3.

\section{Martin integral on $\Delta$}

In this section, we formulate and prove the Martin integral representation (0.4), and then discuss some resulting facts. Let us begin with recalling the bijective correspondence between (0.2) and (0.3). A cylindrical subset of $\mathfrak{T}(\widehat{T})$ (= the set of infinite paths from $\varnothing$ on the branching graph $\mathbb{Y}(\widehat{T}))$ is associated with the finite path $u=(u(0) \nearrow \ldots \nearrow u(n))$ as

$$
C_{u}=\{t \in \mathfrak{T}(\widehat{T}) \mid t(k)=u(k), k=0,1, \ldots, n\} .
$$

Consider the Borel $\sigma$-field $\mathfrak{B}$ generated by the cylindrical subsets of $\mathfrak{T}(\widehat{T})$. Let $\mathcal{P}(\mathfrak{T}(\widehat{T}))$ denote the set of probabilities on measurable space $(\mathfrak{T}(\widehat{T}), \mathfrak{B})$, and $M \in$ $\mathcal{P}(\mathfrak{T}(\widehat{T})$ ) is said to be central (as already anticipated in $(0.3)$ ) if it satisfies

$$
M\left(C_{u}\right) / w_{u}=M\left(C_{v}\right) / w_{v}
$$

for arbitrary finite paths $u$ and $v$ from $\varnothing$ whenever they share a common terminating vertex. Recall (1.21) for the definition of weight $w_{u}$. In the case of a branching graph for wreath product groups, since (1.24) yields $w_{u}=w_{v}$ for these $u$ and $v,(3.1)$ simply reduces to

$$
M\left(C_{u}\right)=M\left(C_{v}\right) .
$$

Let $\mathcal{M}(\mathfrak{T}(\widehat{T}))$ denote the set of central probabilities on $(\mathfrak{T}(\widehat{T}), \mathfrak{B})$ (see $(0.3)$ ). Then, the bijective correspondence between $\varphi \in \mathcal{H}(\mathbb{Y}(\widehat{T}))$ and $M \in \mathcal{M}(\mathfrak{T}(\widehat{T}))$ is given by the equation

$$
\varphi(\Lambda)=\frac{M\left(C_{u}\right)}{w_{u}}, \quad u=(\varnothing \nearrow \cdots \nearrow \Lambda) .
$$

We refer to [9, Section 2.2] for central probabilities and their correspondence to harmonic functions on a general branching graph. As noted there, the centrality of $M \in \mathcal{P}(\mathfrak{T}(\widehat{T}))$ is characterized by a certain invariance of $M$ with respect to a transformation group on $\mathfrak{T}(\widehat{T})$. Then, the ergodicity of $M$ is naturally considered with respect to the transformation group, ${ }^{\dagger}$ and $M$ is ergodic if and only if an invariant subset of $\mathfrak{T}(\widehat{T})$ with respect to the transformation group is either $\varnothing$ or $\mathfrak{T}(\widehat{T})$ up to $M$-measure 0 . By a standard argument in measure theory, this is equivalent to the extremality of $M$ in the set of the central probabilities on $\mathfrak{T}(\widehat{T})$ (see [9, Section 2.2]).

\footnotetext{
$\dagger$ Allow us to make a correction to [9, Section 2.2] here. In [9, pp. 1200-1201], the transformation group on $\mathfrak{T}(\widehat{T})$ mentioned above should be written as $\left\langle\mathfrak{S}_{\mathfrak{T}(\alpha)} \mid \alpha \in \mathbb{G}\right\rangle$, the group generated by $\mathfrak{S}_{\mathfrak{T}(\alpha)}$ 's, instead of $\bigcup_{\alpha \in \mathbb{G}} \mathfrak{S}_{\mathfrak{T}(\alpha)}$ since the inclusion $\mathfrak{S}_{\mathfrak{T}(\alpha)} \subset \mathfrak{S}_{\mathfrak{T}(\beta)}$ does not hold even if $\alpha \nearrow \cdots \nearrow \beta$.
} 
We have that $M \in \mathcal{M}(\mathfrak{T}(\widehat{T}))$ yields the $n$th marginal distribution $M^{(n)}$ on $\mathbb{Y}_{n}(\widehat{T}):$ for $\Lambda \in \mathbb{Y}_{n}(\widehat{T})$

$$
\begin{aligned}
M^{(n)}(\Lambda) & =M(\{t \in \mathfrak{T}(\widehat{T}) \mid t(n)=\Lambda\}) \\
& =\sum_{\text {finite path } u: u(n)=\Lambda} M\left(C_{u}\right)=\sum_{u} w_{u} \varphi(\Lambda)=d(\Lambda) \varphi(\Lambda)
\end{aligned}
$$

by recalling (3.2) and (1.22). Consider the injective map $\iota^{(n)}: \mathbb{Y}_{n}(\widehat{T}) \longrightarrow \Delta$ defined through the rescaled Frobenius coordinates by

$$
\Lambda=\left(\lambda^{\zeta}\right)_{\zeta \in \widehat{T}} \in \mathbb{Y}_{n}(\widehat{T}) \longmapsto\left(\left(\frac{a_{i}\left(\lambda^{\zeta}\right)}{n}\right)_{\zeta, i},\left(\frac{b_{i}\left(\lambda^{\zeta}\right)}{n}\right)_{\zeta, i},\left(\frac{\left|\lambda^{\zeta}\right|}{n}\right)_{\zeta}\right) \in \Delta .
$$

Recall that $+1 / 2$ is added in (2.18), which ensures that the image of $\iota^{(n)}$ is included in $\Delta$.

\section{THEOREM 3.1}

Any $\varphi \in \mathcal{H}(\mathbb{Y}(\widehat{T}))$ yields an integral representation

$$
\varphi(\Lambda)=\int_{\Delta} K(\Lambda, \omega) Q(d \omega), \quad \Lambda \in \mathbb{Y}(\widehat{T}) .
$$

Here kernel $K(\Lambda, \omega)$ is given by (2.28), and $Q \in \mathcal{P}(\Delta)$ is uniquely determined by $\varphi$. Taking $M \in \mathcal{M}(\mathfrak{T}(\widehat{T}))$ corresponding to $\varphi$ through (3.2), we have that

$$
Q=\lim _{n \rightarrow \infty} \iota_{*}^{(n)} M^{(n)}
$$

as a weakly converging limit in $\mathcal{P}(\Delta)$.

\section{REMARK 3.2}

We have that $\widetilde{\Delta}$ is equipped with the metrizable topology of the product of the pointwise converging one mentioned after (2.24), which is separable, and also with the associated Borel structure. Additionally, $\Delta$ is a Borel subset of $\widetilde{\Delta}$. Recall that if $S$ is a separable metric space, then $\mathcal{P}(S)$ is equipped with a separable metrizable topology characterized by weak convergence of a sequence of probabilities:

$$
\lim _{n \rightarrow \infty} \int_{S} f(s) \mu_{n}(d s)=\int_{S} f(s) \mu(d s),
$$

for any bounded continuous function $f$ on $S$. The procedure of (3.6) taking a weak limit after pushing forward the marginal distribution to the boundary seems to be analogous to the classical radial limit for a harmonic function on the unit disk.

\section{Proof}

Step 1. First we show the uniqueness of $Q \in \mathcal{P}(\Delta)$ in (3.5). Let $\mathcal{S}$ be the set of $\mathbb{R}$-linear combinations of $\{K(\Lambda, \cdot) \mid \Lambda \in \mathbb{Y}(\widehat{T})\}$ in (2.28). We verify that $\mathcal{S}$ is dense in $C(\Delta ; \mathbb{R})$ for finite $T$ and in $C(\widetilde{\Delta} ; \mathbb{R})$ for infinite $T$ by using the StoneWeierstrass theorem. In fact, $\mathcal{S}$ contains the constant 1 , which corresponds to $\Lambda=\varnothing$. Lemma 2.3 ensures that the $p_{k}^{\zeta}$ 's and hence the $s_{\lambda}^{\zeta}$ 's also separate two arbi- 
trary points in $\Delta$ or $\widetilde{\Delta}$. By (2.27), the $\mathbb{R}$-linear combinations of $\left\{s_{\lambda}^{\zeta}\right\}_{\lambda \in \mathbb{Y}}$ coincide with those of $\left\{p_{\rho}^{\zeta}\right\}_{\rho \in \mathbb{Y}}$, the latter clearly forming an algebra. Hence $K(\Lambda, \cdot) K(\mathrm{M}, \cdot)$ for $\Lambda, \mathrm{M} \in \mathbb{Y}(\widehat{T})$ is linearized and belongs to $\mathcal{S}$. This completes the proof of the density of $\mathcal{S}$. Then, integration on $\mathcal{S}$ determines that on the whole $C(\Delta ; \mathbb{R})$ or $C(\widetilde{\Delta} ; \mathbb{R})$. Thus, $Q \in \mathcal{P}(\Delta)$ or $\mathcal{P}(\widetilde{\Delta})$ is uniquely determined. Note that if $T$ is infinite, then an element of $\mathcal{P}(\widetilde{\Delta})$ supported by $\Delta$ is identified with that of $\mathcal{P}(\Delta)$.

Step 2. We show that if there exists a limit

$$
Q_{0}=\lim _{n \rightarrow \infty} \iota_{*}^{(n)} M^{(n)}
$$

in $\mathcal{P}(\Delta)$ for finite $T$ or in $\mathcal{P}(\widetilde{\Delta})$ for infinite $T$, then $\varphi$ is expressed as

$$
\varphi(\Lambda)=\int_{\Delta} K(\Lambda, \omega) Q_{0}(d \omega) \quad \text { or } \quad \varphi(\Lambda)=\int_{\widetilde{\Delta}} K(\Lambda, \omega) Q_{0}(d \omega)
$$

for $\Lambda \in \mathbb{Y}(\widehat{T})$. Let $T$ be infinite, since the case of finite $T$ is treated in the same way. Combining

$$
\varphi(\Lambda)=\sum_{\mathrm{N} \in \mathbb{Y}_{n}(\widehat{T})} d(\Lambda, \mathrm{N}) \varphi(\mathrm{N})=\sum_{\mathrm{N} \in \mathbb{Y}_{n}(\widehat{T})} K(\Lambda, \mathrm{N}) M^{(n)}(\mathrm{N}),
$$

which is seen from (3.3), and

$$
\int_{\widetilde{\Delta}} K(\Lambda, \omega) \iota_{*}^{(n)} M^{(n)}(d \omega)=\sum_{\mathrm{N} \in \mathbb{Y}_{n}(\widehat{T})} K\left(\Lambda, \iota^{(n)} \mathrm{N}\right) M^{(n)}(\mathrm{N})
$$

we write

$$
\begin{aligned}
& \left|\varphi(\Lambda)-\int_{\widetilde{\Delta}} K(\Lambda, \omega) Q_{0}(d \omega)\right| \\
& \leqq\left|\varphi(\Lambda)-\int_{\widetilde{\Delta}} K(\Lambda, \omega) \iota_{*}^{(n)} M^{(n)}(d \omega)\right| \\
& +\left|\int_{\widetilde{\Delta}} K(\Lambda, \omega) \iota_{*}^{(n)} M^{(n)}(d \omega)-\int_{\widetilde{\Delta}} K(\Lambda, \omega) Q_{0}(d \omega)\right| \\
& \leqq \sum_{\mathrm{N} \in \mathbb{Y}_{n}(\widehat{T})}\left|K(\Lambda, \omega)-K\left(\Lambda, \iota^{(n)} \mathrm{N}\right)\right| M^{(n)}(\mathrm{N}) \\
& +\left|\int_{\widetilde{\Delta}} K(\Lambda, \omega) \iota_{*}^{(n)} M^{(n)}(d \omega)-\int_{\widetilde{\Delta}} K(\Lambda, \omega) Q_{0}(d \omega)\right| .
\end{aligned}
$$

Comparing (2.3) with

$$
\begin{aligned}
K\left(\Lambda, \iota^{(n)} \mathrm{N}\right) & =\prod_{\zeta \in \widehat{T}} \frac{1}{(\operatorname{dim} \zeta)^{|\lambda \zeta|}} s_{\lambda \zeta}^{\zeta}\left(\iota^{(n)} \mathrm{N}\right) \\
& =\prod_{\zeta \in \widehat{T}} \frac{1}{(\operatorname{dim} \zeta)^{|\lambda \zeta|}} \sum_{\rho \in \mathbb{Y}_{|\lambda \zeta|}} \frac{1}{z_{\rho}} \chi_{\rho}^{\lambda} p_{\rho}^{\zeta}\left(\left(\frac{a_{i}\left(\nu^{\zeta}\right)}{n}\right)_{\zeta, i},\left(\frac{b_{i}\left(\nu^{\zeta}\right)}{n}\right)_{\zeta, i},\left(\frac{\left|\nu^{\zeta}\right|}{n}\right)_{\zeta}\right) \\
& =\prod_{\zeta \in \widehat{T}} \frac{1}{(\operatorname{dim} \zeta)^{|\lambda \zeta|}} \sum_{\rho \in \mathbb{Y}_{|\lambda \zeta|}} \frac{1}{z_{\rho}} \chi_{\rho}^{\lambda} \frac{1}{n^{|\rho|}} p_{\rho}\left(\nu^{\zeta}\right)
\end{aligned}
$$


and using the estimate of (2.31), we have

$$
\left|K(\Lambda, \mathrm{N})-K\left(\Lambda, \iota^{(n)} \mathrm{N}\right)\right| \leqq \frac{1}{n} C_{k}, \quad \Lambda \in \mathbb{Y}_{k}(\widehat{T}), \mathrm{N} \in \mathbb{Y}_{n}(\widehat{T})
$$

Hence the first term on the rightmost side of (3.8) is bounded by $C_{k} / n$. The second term converges to 0 as $n \rightarrow \infty$ since $K(\Lambda, \cdot) \in C(\widetilde{\Delta} ; \mathbb{R})$. We thus obtain (3.7).

Step 3. Since $\mathcal{P}(\Delta)$ for finite $T$ and $\mathcal{P}(\widetilde{\Delta})$ for infinite $T$ are compact (and metrizable) with respect to the topology of weak convergence, $\left\{\iota_{*}^{(n)} M^{(n)}\right\}_{n \in \mathbb{N}}$ contains a converging subsequence. The argument in Step 2 implies that its limit $Q_{0}$ satisfies (3.7) and hence is unique. Hence the whole sequence $\left\{\iota_{*}^{(n)} M^{(n)}\right\}_{n \in \mathbb{N}}$ converges as $n \rightarrow \infty$. This completes the proof in the case of finite $T$. If $T$ is infinite, then we have

$$
Q=\lim _{n \rightarrow \infty} \iota_{*}^{(n)} M^{(n)} \quad \text { in } \mathcal{P}(\widetilde{\Delta}) \quad \text { and } \quad \varphi(\Lambda)=\int_{\widetilde{\Delta}} K(\Lambda, \omega) Q(d \omega) .
$$

Using the harmonicity of $\varphi$, we have

$$
\begin{aligned}
0 & =\varphi(\Lambda)-\sum_{\mathrm{N}: \Lambda \nearrow \mathrm{N}} \kappa(\Lambda, \mathrm{N}) \varphi(\mathrm{N}) \\
& =\int_{\widetilde{\Delta}} K(\Lambda, \omega) Q(d \omega)-\sum_{\mathrm{N}: \Lambda \nearrow \mathrm{N}} \kappa(\Lambda, \mathrm{N}) \int_{\widetilde{\Delta}} K(\mathrm{~N}, \omega) Q(d \omega) \\
& =\int_{\widetilde{\Delta}}\left(K(\Lambda, \omega)-\sum_{\mathrm{N}: \Lambda \nearrow \mathrm{N}} \kappa(\Lambda, \mathrm{N}) K(\mathrm{~N}, \omega)\right) Q(d \omega) .
\end{aligned}
$$

The superharmonicity of $K(\cdot, \omega)$ (Lemma 2.7) then yields

$$
K(\Lambda, \omega)=\sum_{\mathrm{N}: \Lambda \nearrow \mathrm{N}} \kappa(\Lambda, \mathrm{N}) K(\mathrm{~N}, \omega), \quad Q \text {-a.e. } \omega .
$$

Taking a common exceptional set of $Q$-measure 0 for $\Lambda \in \mathbb{Y}(\widehat{T})$ in (3.9), we verify that $K(\cdot, \omega)$ is harmonic on $\mathbb{Y}(\widehat{T})$ for almost every $\omega$. In other words, $Q$ is supported by $\Delta$ and regarded as an element of $\mathcal{P}(\Delta)$. We note that

$$
Q=\lim _{n \rightarrow \infty} \iota_{*}^{(n)} M^{(n)} \quad \text { in } \mathcal{P}(\widetilde{\Delta}) \quad \text { and } \quad \iota_{*}^{(n)} M^{(n)}, Q \in \mathcal{P}(\Delta)
$$

imply the convergence in $\mathcal{P}(\Delta)$, which is seen, for example, from the characterization of weak convergence of probabilities in terms of the convergence on any Borel set with boundary of probability 0 .

Recall that the minimal Martin boundary is defined in (1.35) as

$$
\partial_{m} \mathbb{Y}(\widehat{T})=\{\omega \in \partial \mathbb{Y}(\widehat{T}) \mid K(\cdot, \omega) \text { is minimal harmonic }\} .
$$

For $\omega \in \partial \mathbb{Y}(\widehat{T}), K(\varnothing, \omega)=1$ and $K(\Lambda, \omega) \geqq 0$ hold. Letting ex $C$ denote the set of extremal points of convex set $C$, we always have

$$
\left\{K(\cdot, \omega) \mid \omega \in \partial_{m} \mathbb{Y}(\widehat{T})\right\} \subset \operatorname{ex} \mathcal{H}(\mathbb{Y}(\widehat{T})) .
$$

On the other hand, the Martin integral representation (Theorem 3.1) ensures that 


$$
\operatorname{ex} \mathcal{H}(\mathbb{Y}(\widehat{T})) \subset\{K(\cdot, \omega) \mid \omega \in \Delta\}
$$

Actually, we have equalities in (3.10) and (3.11) as follows.

\section{THEOREM 3.3}

An extremal point of $\mathcal{H}(\mathbb{Y}(\widehat{T}))$ is exactly a Martin kernel $K(\cdot, \omega)$ for $\omega \in \Delta$. The minimal Martin boundary $\partial_{m} \mathbb{Y}(\widehat{T})$ of the branching graph $\mathbb{Y}(\widehat{T})$ is homeomorphically isomorphic to $\Delta$ for any $T$.

Proof

Let $\omega \in \Delta$. By Lemma 2.8, $K(\cdot, \omega)$ is harmonic. If it is expressed as a convex combination in $\mathcal{H}(\mathbb{Y}(\widehat{T}))$, then

$$
K(\cdot, \omega)=c \varphi_{1}+(1-c) \varphi_{2}, \quad \varphi_{1}, \varphi_{2} \in \mathcal{H}(\mathbb{Y}(\widehat{T})), 0 \leqq c \leqq 1 .
$$

Theorem 3.1 applied to $\varphi_{1}$ and $\varphi_{2}$ yields

$$
K(\Lambda, \omega)=c \int_{\Delta} K\left(\Lambda, \omega^{\prime}\right) Q_{1}\left(d \omega^{\prime}\right)+(1-c) \int_{\Delta} K\left(\Lambda, \omega^{\prime}\right) Q_{2}\left(d \omega^{\prime}\right), \quad \Lambda \in \mathbb{Y}(\widehat{T}),
$$

for $Q_{1}, Q_{2} \in \mathcal{P}(\Delta)$. The uniqueness part of Theorem 3.1 implies that

$$
\delta_{\omega}=c Q_{1}+(1-c) Q_{2}
$$

holds in $\mathcal{P}(\Delta)$, which is possible only when $c=0$ or $c=1$ or $Q_{1}=Q_{2}$. This shows the extremality $(=$ minimality) of $K(\cdot, \omega)$ in $\mathcal{H}(\mathbb{Y}(\widehat{T}))$; in other words,

$$
\{K(\cdot, \omega) \mid \omega \in \Delta\} \subset\left\{K(\cdot, \omega) \mid \omega \in \partial_{m} \mathbb{Y}(\widehat{T})\right\} .
$$

Combining (3.12) with (3.10) and (3.11), we have equality between them. The homeomorphic property is already shown in Theorem 2.5.

Combining Theorems 2.5 and 3.3, we can translate the obtained results into those for the other objects in (a.2)-(a.4) described in the introduction. Recall the bijective correspondence

$$
f \in \mathcal{K}\left(\mathfrak{S}_{\infty}(T)\right) \longleftrightarrow \varphi \in \mathcal{H}(\mathbb{Y}(\widehat{T}))
$$

given by

$$
\left.f\right|_{\mathfrak{S}_{k}(T)}=\sum_{\Lambda \in \mathbb{Y}_{k}(\widehat{T})} \varphi(\Lambda) \chi^{\Lambda}, \quad k \in \mathbb{N}
$$

An extremal point of $\mathcal{K}\left(\mathfrak{S}_{\infty}(T)\right)$ is simply called a character of $\mathfrak{S}_{\infty}(T)$. It is known that a character of $\mathfrak{S}_{\infty}(T)$ is factorizable (or multiplicative) with respect to the standard decomposition (1.1) into basic elements. After showing such a factorizability, (3.14) of Theorem 3.4 for a character of $\mathfrak{S}_{\infty}(T)$ was first obtained by the second author and E. Hirai (see [4] and [5]). In this paper, however, we compute a character of $\mathfrak{S}_{\infty}(T)$ directly by using (2.28) under the correspondence of Theorem 3.3 without knowing a priori the factorizability. The structure of the conjugacy classes of $\mathfrak{S}_{\infty}(T)$ is given by (1.15). 


\section{THEOREM 3.4}

Let $f$ be a character of $\mathfrak{S}_{\infty}(T)$, and let $\omega \in \Delta$ be the corresponding parameter in Theorem 3.3. The value of $f=f_{\omega}$ at an element in the conjugacy class of $\mathfrak{S}_{\infty}(T)$ corresponding to $\mathrm{P}=\left(\rho_{\theta}\right)_{\theta \in[T]} \in \mathbb{Y}([T])$, denoted by $f_{\omega}(\mathrm{P})$, is given by

$$
f_{\omega}(\mathrm{P})=\prod_{j=1}^{\infty} \prod_{\theta \in[T]}\left(\sum_{\zeta \in \widehat{T}} p_{j}^{\zeta}(\omega) \frac{\chi_{\theta}^{\zeta}}{(\operatorname{dim} \zeta)^{j}}\right)^{m_{j}\left(\rho_{\theta}\right)} .
$$

In particular, $f$ is factorizable.

Proof

Take $x \in C_{\mathrm{P}} \subset \mathfrak{S}_{\infty}(T)$, and then take $k \in \mathbb{N}$ such that $x \in \mathfrak{S}_{k}(T)$. Let $\operatorname{supp} \mathrm{P}=$ $\left\{\theta_{1}, \theta_{2}, \ldots, \theta_{l}\right\}$, where supp P denotes the set of $\theta$ 's with nontrivial $\rho_{\theta}$ 's. For computing $f_{\omega}(\mathrm{P})$ through (3.13), we have formulas for $\varphi(\Lambda)=K(\Lambda, \omega)$ in $(2.28)$ and for $\chi^{\Lambda}$ in (1.12) (in the case of $k=n$ ). Noting that the condition (1.11) reduces to

$$
\sum_{\left(\rho_{\theta_{i}}\right)_{j}^{*}: r\left(\left(\rho_{\theta_{i}}\right)_{j}^{*}\right)=\zeta}\left(\rho_{\theta_{i}}\right)_{j}=\left|\lambda^{\zeta}\right|, \quad \zeta \in \widehat{T},
$$

we have

$$
\begin{aligned}
f_{\omega}(\mathrm{P})= & \sum_{\Lambda \in \mathbb{Y}_{k}(\widehat{T})} \sum_{r \text { satisfying }} \prod_{(3.15)}\left\{\frac{1}{(\operatorname{dim} \zeta)^{\left|\lambda^{\zeta}\right|}}\right. \\
& \left.\times s_{\lambda^{\zeta}}^{\zeta}(\omega)\left(\prod_{i=1}^{l}\left(\chi_{\theta_{i}}^{\zeta}\right)^{l\left(r^{-1}(\zeta) \cap \rho_{\theta_{i}}\right)}\right) \chi_{r^{-1}(\zeta)}^{\lambda^{\zeta}}\right\} .
\end{aligned}
$$

In (3.16), let $\Lambda=\left(\lambda^{\zeta}\right)_{\zeta \in \widehat{T}}$ range over $\mathbb{Y}_{k}(\widehat{T})$ in two stages. First, take the finite subset $F$ of $\widehat{T}$ as $\operatorname{supp} \Lambda$, that is, the $\zeta$ 's with nontrivial $\lambda^{\zeta}$ 's, and then take $n^{\zeta} \in \mathbb{N}$ for $\zeta \in F$ as $n^{\zeta}=\left|\lambda^{\zeta}\right|$. We necessarily have that $\sum_{\zeta \in F} n^{\zeta}=k$. Next, for each $\left(F,\left(n^{\zeta}\right)_{\zeta \in F}\right)$, let $\Lambda \in \mathbb{Y}_{k}(\widehat{T})$ range under the constraint of

$$
\operatorname{supp} \Lambda=F \quad \text { and } \quad\left|\lambda^{\zeta}\right|=n^{\zeta} .
$$

For fixed $\left(F,\left(n^{\zeta}\right)_{\zeta \in F}\right)$, note that the sum in $\Lambda$ in the above second stage and the sum $\sum_{r}$ in (3.16) commute. Setting the condition

$$
\sum_{\left(\rho_{\theta_{i}}\right)_{j}^{*}: r\left(\left(\rho_{\theta_{i}}\right)_{j}^{*}\right)=\zeta}\left(\rho_{\theta_{i}}\right)_{j}=n^{\zeta}, \quad \zeta \in \widehat{T},
$$

instead of (3.15), we hence continue (3.16) as

$$
\begin{aligned}
(3.16)= & \sum_{\left(F,\left(n^{\zeta}\right)_{\zeta \in F}\right)} \sum_{r:(3.18)} \sum_{\Lambda:(3.17)} \prod_{\zeta \in F}\left\{\frac{1}{(\operatorname{dim} \zeta)^{n^{\zeta}}}\right. \\
& \left.\times\left(\prod_{i=1}^{l}\left(\chi_{\theta_{i}}^{\zeta}\right)^{l\left(r^{-1}(\zeta) \cap \rho_{\theta_{i}}\right)}\right) \chi_{r^{-1}(\zeta)}^{\lambda^{\zeta}} s_{\lambda \zeta}^{\zeta}(\omega)\right\} \\
= & \sum_{\left(F,\left(n^{\zeta}\right)_{\zeta \in F}\right)} \sum_{r:(3.18)} \prod_{\zeta \in F}\left\{p_{r^{-1}(\zeta)}^{\zeta} \frac{1}{(\operatorname{dim} \zeta)^{n^{\zeta}}}\left(\prod_{i=1}^{l}\left(\chi_{\theta_{i}}^{\zeta}\right)^{l\left(r^{-1}(\zeta) \cap \rho_{\theta_{i}}\right)}\right)\right\}
\end{aligned}
$$


by using the inversion of (2.27). Let us verify that this expression is equal to

$$
\prod_{i=1}^{l} \prod_{j=1}^{\infty}\left(\sum_{\zeta \in \widehat{T}} p_{j}^{\zeta}(\omega) \frac{1}{(\operatorname{dim} \zeta)^{j}} \chi_{\theta_{i}}^{\zeta}\right)^{m_{j}\left(\rho_{\theta_{i}}\right)}
$$

In (3.20), since two kinds of products are finite ones and the infinite sum in $\zeta$ converges absolutely, no problems occur in changing the terms. Let $n^{\zeta}$ be the degree of the factors labeled by $\zeta$ in each term of the development of (3.20), where $p_{j}^{\zeta}(\omega)$ is regarded to have degree $j$. We have

$$
\sum_{\zeta \in \widehat{T}} n^{\zeta}=\sum_{i=1}^{l} \sum_{j=1}^{\infty} j m_{j}\left(\rho_{\theta_{i}}\right)=\sum_{i=1}^{l}\left|\rho_{\theta_{i}}\right|=k .
$$

For a term in the development of (3.20), let $F$ be the $\zeta$ 's which actually appear, and let $\left(n^{\zeta}\right)_{\zeta \in F}$ be the set of degrees labeled by $\zeta$. Given $\left(F,\left(n^{\zeta}\right)_{\zeta \in F}\right)$, the corresponding terms in (3.20) are counted by the maps $r$ satisfying (3.18). We then observe that the term corresponding to $\left(F,\left(n^{\zeta}\right)_{\zeta \in F}, r\right)$ is expressed in a product form seen in the rightmost side of (3.19). This completes the proof of (3.14).

\section{THEOREM 3.5}

Any $f \in \mathcal{K}\left(\mathfrak{S}_{\infty}(T)\right)$ yields an integral representation

$$
f(\mathrm{P})=\int_{\Delta} f_{\omega}(\mathrm{P}) Q(d \omega), \quad \mathrm{P} \in \mathbb{Y}([T])
$$

Here the character $f_{\omega}$ is given by (3.14), and $Q \in \mathcal{P}(\Delta)$ is the same as that of Theorem 3.1 under the correspondence $f \in \mathcal{K}\left(\mathfrak{S}_{\infty}(T)\right) \leftrightarrow \varphi \in \mathcal{H}(\mathbb{Y}(\widehat{T}))$ through (3.13).

Proof

For given $\mathrm{P} \in \mathbb{Y}([T])$, take $x \in C_{\mathrm{P}} \subset \mathfrak{S}_{\infty}(T)$, and then take $k \in \mathbb{N}$ such that $x \in$ $\mathfrak{S}_{k}(T)$. The integral representation for an element of $\mathcal{H}(\mathbb{Y}(\widehat{T})$ ) (see Theorem 3.1) is easily transformed to the one for the corresponding element of $\mathcal{K}\left(\mathfrak{S}_{\infty}(T)\right)$. More precisely, we have

$$
\begin{aligned}
f(x) & =\sum_{\Lambda \in \mathbb{Y}_{k}(\widehat{T})} \varphi(\Lambda) \chi^{\Lambda}(x)=\sum_{\Lambda \in \mathbb{Y}_{k}(\widehat{T})}\left(\int_{\Delta} K(\Lambda, \omega) Q(d \omega)\right) \chi^{\Lambda}(x) \\
& =\int_{\Delta}\left(\sum_{\Lambda \in \mathbb{Y}_{k}(\widehat{T})} K(\Lambda, \omega) \chi^{\Lambda}(x)\right) Q(d \omega) \\
& =\int_{\Delta} f_{\omega}(\mathrm{P}) Q(d \omega),
\end{aligned}
$$

where changing the sum and integral is justified by

$$
\sum_{\Lambda \in \mathbb{Y}_{k}(\widehat{T})} \int_{\Delta}\left|K(\Lambda, \omega) \chi^{\Lambda}(x)\right| Q(d \omega) \leqq \sum_{\Lambda \in \mathbb{Y}_{k}(\widehat{T})} d(\Lambda) \varphi(\Lambda)=1 .
$$




\section{Characters, harmonic functions, and central probabilities associated with an inductive system of compact groups}

We note bijective correspondences between the spaces of (0.1)-(0.3) for an inductive system of compact groups instead of restricting to wreath products $\mathfrak{S}_{n}(T)$. Let us consider a sequence of compact groups

$$
\{e\}=G_{0} \subset G_{1} \subset \cdots \subset G_{n-1} \subset G_{n} \subset \cdots
$$

in which the embedding $G_{n-1} \subset G_{n}$ is a continuous homomorphism for any $n \in \mathbb{N}$. Set $G_{\infty}=\lim _{n \rightarrow \infty} G_{n}=\bigcup_{n=0}^{\infty} G_{n}$ (inductive limit group). Letting $\widehat{G}_{n}$ denote the set of equivalence classes of IURs of $G_{n}$, we have a branching graph with $\mathbb{G}=\bigsqcup_{n=0}^{\infty} \widehat{G}_{n}$ as the vertex set by introducing edge $\alpha \nearrow \beta$ and its multiplicity $\kappa(\alpha, \beta)$ for $\alpha \in \widehat{G}_{n-1}$ and $\beta \in \widehat{G}_{n}$ through the irreducible decomposition

$$
\operatorname{Res}_{G_{n-1}}^{G_{n}} \pi^{\beta} \cong \bigoplus_{\alpha \in \widehat{G}_{n-1}: \alpha \nearrow \beta} \kappa(\alpha, \beta) \pi^{\alpha}, \quad \pi^{\beta} \in \beta .
$$

The case of $G_{n}=\mathfrak{S}_{n}(T)$ was treated in Section 1.8.

Until the end of the proof of Proposition 4.2, we stop assuming that $\widehat{G}_{n}$ is countable.

Equipping $G_{\infty}$ with the inductive limit topology, set

$$
\begin{aligned}
\mathcal{K}\left(G_{\infty}\right)= & \left\{f: G_{\infty} \longrightarrow \mathbb{C} \mid\right. \\
& f \text { is continuous, positive definite, and central, } f(e)=1\} .
\end{aligned}
$$

We modify (0.2) by adding "countably supported" and set

$$
\begin{aligned}
\mathcal{H}(\mathbb{G})= & \{\varphi: \mathbb{G} \longrightarrow \mathbb{C} \mid \varphi \text { is harmonic and nonnegative, } \varphi(\varnothing)=1, \\
& \operatorname{supp} \varphi \text { is an at most countable set }\} .
\end{aligned}
$$

Here the harmonicity of $\varphi$ is defined by

$$
\varphi(\alpha)=\sum_{\beta: \alpha \nearrow \beta} \kappa(\alpha, \beta) \varphi(\beta), \quad \alpha \in \mathbb{G},
$$

similarly to (1.18). If a graph consisting of the subset $\mathbb{G}^{0} \subset \mathbb{G}$ and the edges inherited from $\mathbb{G}$ satisfies that, for any $\beta \in \mathbb{G}^{0}$, all vertices lying on the finite paths from $\varnothing$ to $\beta$ belong to $\mathbb{G}^{0}$, then we refer to $\mathbb{G}^{0}$ simply as a subgraph of $\mathbb{G}$. It is immediate that $\operatorname{supp} \varphi$ is a subgraph of $\mathbb{G}$ for harmonic and nonnegative $\varphi$. Let $\mathfrak{T}(\mathbb{G})$ denote the set of all infinite paths $t=(t(0) \nearrow t(1) \nearrow t(2) \nearrow \cdots), t(n) \in$ $\widehat{G}_{n}$, on branching graph $\mathbb{G} ; \mathfrak{T}(\mathbb{G})$ is equipped with the $\sigma$-field generated by its cylindrical subsets. We consider $\mathfrak{T}\left(\mathbb{G}^{0}\right)$ also for the subgraph $\mathbb{G}^{0} \subset \mathbb{G}$, where $t(n) \in \mathbb{G}_{n}^{0}=\mathbb{G}^{0} \cap \widehat{G}_{n}$. We modify $(0.3)$ to set

$$
\begin{aligned}
\mathcal{M}(\mathfrak{T}(\mathbb{G}))= & \left\{M \in \mathcal{P}(\mathfrak{T}(\mathbb{G})) \mid M \text { is central and supported by } \mathfrak{T}\left(\mathbb{G}^{0}\right)\right. \\
& \text { for some countable subgraph } \left.\mathbb{G}^{0} \subset \mathbb{G}\right\} .
\end{aligned}
$$

Here the centrality of $M$ is defined similarly to (3.1) by

$$
M\left(C_{u}\right) / w_{u}=M\left(C_{v}\right) / w_{v}
$$


for finite paths $u$ and $v$ from $\varnothing$ terminating at a common vertex, where $w_{u}$ is the weight of path $u$ defined by (1.21) and $C_{u}$ is the cylindrical set associated with $u$. Note that $\mathcal{K}\left(G_{\infty}\right), \mathcal{H}(\mathbb{G})$, and $\mathcal{M}(\mathfrak{T}(\mathbb{G}))$ are all convex sets.

\section{PROPOSITION 4.1}

There exist bijective affine maps between $\mathcal{K}\left(G_{\infty}\right), \mathcal{H}(\mathbb{G})$, and $\mathcal{M}(\mathfrak{T}(\mathbb{G}))$. In particular, their extremal points have bijective correspondences.

Proof

Recall that the function $a: C_{1} \rightarrow C_{2}$, where $C_{1}$ and $C_{2}$ are convex sets, is said to be affine if it satisfies $a(s x+(1-s) y)=s a(x)+(1-s) a(y)$ for any $x, y \in C_{1}$ and $0 \leqq s \leqq 1$. A bijection between $\mathcal{H}(\mathbb{G})$ and $\mathcal{M}(\mathfrak{T}(\mathbb{G}))$ is given in the same way with (3.2): for $\varphi \in \mathcal{H}(\mathbb{G})$ and $M \in \mathcal{M}(\mathfrak{T}(\mathbb{G}))$,

$$
\varphi(\alpha)=M\left(C_{u}\right) / w_{u}, \quad \alpha \in \mathbb{G},
$$

where $\alpha$ is the terminal vertex of finite path $u$. This fact is fully shown in $[9$, Lemma 2.9] for a general branching graph.

As noted in (3.13), a bijection between $\mathcal{K}\left(G_{\infty}\right)$ and $\mathcal{H}(\mathbb{G})$ is given by using Fourier expansion on each compact group $G_{k}$ : for $f \in \mathcal{K}\left(G_{\infty}\right)$ and $\varphi \in \mathcal{H}(\mathbb{G})$,

$$
\left.f\right|_{G_{k}}=\sum_{\alpha \in \widehat{G}_{k}} \varphi(\alpha) \chi^{\alpha}, \quad k \in \mathbb{N},
$$

where $\chi^{\alpha}$ denotes the irreducible character for $\alpha ; \chi^{\alpha}(x)=\operatorname{tr} \pi^{\alpha}(x)$. This fact is shown in [9, Theorem 4.2] for $G_{n}=\mathfrak{S}_{n}(T)$ with an arbitrary compact group $T$. No modification is needed in its proof to apply it to general $G_{n}$. Either bijection given by (4.4) or (4.5) is clearly affine.

The set of extremal elements in $\mathcal{K}\left(G_{\infty}\right)$ is denoted by $\mathcal{E}\left(G_{\infty}\right)$, whose elements are called characters of $G_{\infty}$. Any character of $G_{\infty}$ is approximated by the irreducible characters of $G_{n}$ as will be seen in Proposition 4.2. This implies that not only the extremal elements of $\mathcal{K}\left(G_{\infty}\right)$ but also the ones of $\mathcal{H}(\mathbb{G})$ and $\mathcal{M}(\mathfrak{T}(\mathbb{G}))$ have approximations by the objects at finite level $n$.

\section{PROPOSITION 4.2}

Let $f$ be a character of $G_{\infty}$, and let $M$ be the corresponding probability in $\operatorname{ex} \mathcal{M}(\mathfrak{T}(\mathbb{G})$ ) determined in Proposition 4.1. For $M$-almost sure path $t \in \mathfrak{T}(\mathbb{G})$, the convergence of the normalized irreducible characters

$$
\lim _{n \rightarrow \infty} \tilde{\chi}^{t(n)}=f
$$

holds uniformly on each $G_{k}, k \in \mathbb{N}$.

\section{Proof}

This fact is shown in [9, Theorem 4.3] for $G_{k}=\mathfrak{S}_{k}(T)$ with an arbitrary compact group $T$. No modification is needed in its proof to apply it to general $G_{k}$. With $\varphi \in \operatorname{ex} \mathcal{H}(\mathbb{G})$ being taken correspondingly, the proof is based on the $M$-almost 
sure convergence

$$
\varphi(\alpha)=\lim _{n \rightarrow \infty} K(\alpha, t(n)), \quad \alpha \in \operatorname{supp} \varphi(\text { subgraph of } \mathbb{G}),
$$

which is shown via the martingale convergence theorem in [9, Theorem 3.2].

Let us look at the topologies given the convex sets (4.1), (4.2), and (4.3). Hereafter again, we assume that each $\widehat{G}_{n}$ is at most countable for technical conveniences. Generally speaking, we consider compact-open topology for a space of functions and weak convergence topology for a space of probabilities. To deal with $\mathcal{K}\left(G_{\infty}\right)$, recall that any compact set $K$ of $G_{\infty}$, which is given the inductive limit topology, is included in $G_{n}$ for sufficiently large $n$. (See [8, Section 6.3] for this fact and other properties of an inductive limit topology.) Hence the compact-open topology, or topology of uniform convergence on every compact set, on $\mathcal{K}\left(G_{\infty}\right)$ admits

$$
\left\{\left\{g \in \mathcal{K}\left(G_{\infty}\right)\left|\max _{x \in G_{n}}\right| f(x)-g(x) \mid<\epsilon_{k}\right\} \mid n, k \in \mathbb{N}\right\}
$$

as a fundamental system of neighborhoods of $f$, where $\epsilon_{k}>0$ and $\epsilon_{k} \searrow 0$ as $k \rightarrow \infty$. Since $\mathbb{G}=\bigsqcup_{n=0}^{\infty} \widehat{G}_{n}$ is discrete, the compact-open topology on $\mathcal{H}(\mathbb{G})$ is just the one of pointwise converging on $\mathbb{G}$. The path space $\mathfrak{T}(\mathbb{G})$ is equipped with the relative topology of $\prod_{n=0}^{\infty} \widehat{G}_{n}$ (having the product (or weak) topology of discrete ones on $\widehat{G}_{n}$ ). Since $\mathfrak{T}(\mathbb{G})$ is a separable metric space, by virtue of the countability assumption for $\widehat{G}_{n}$ 's, so is $\mathcal{P}(\mathfrak{T}(\mathbb{G})$ ) with respect to the topology of weak convergence of a sequence of probabilities. (Recall Remark 3.2.) The topology on $\mathfrak{T}(\mathbb{G})$ is also generated by cylindrical sets $\left\{C_{u} \mid u\right.$ : finite path on $\left.\mathbb{G}\right\}$.

LEMMA 4.3

Assume that $\widehat{G}_{n}$ is at most countable for any $n \in \mathbb{N}$. The bijective map

$$
\mathcal{M}(\mathfrak{T}(\mathbb{G})) \longrightarrow \mathcal{H}(\mathbb{G})
$$

induced by the correspondence through (4.4) between $M \in \mathcal{M}(\mathfrak{T}(\mathbb{G}))$ and $\varphi \in$ $\mathcal{H}(\mathbb{G})$ is a homeomorphism.

\section{Proof}

Note that a cylindrical set $C_{u}$ is open and closed. Then, since $1_{C_{u}}$ is a bounded continuous function on $\mathfrak{T}(\mathbb{G})$, the weak convergence of $\left\{M_{n}\right\}_{n \in \mathbb{N}}$ to $M$ in $\mathcal{P}(\mathfrak{T}(\mathbb{G})$ ) implies convergence on every cylindrical set.

To show continuity of the inverse map $\mathcal{H}(\mathbb{G}) \longrightarrow \mathcal{M}(\mathfrak{T}(\mathbb{G}))$, let a sequence $\left\{\varphi_{n}\right\}$ converge to $\varphi$ in $\mathcal{H}(\mathbb{G})$. Take $M_{n} \in \mathcal{M}(\mathfrak{T}(\mathbb{G}))$ and $M \in \mathcal{M}(\mathfrak{T}(\mathbb{G}))$ corresponding to $\varphi_{n}$ and $\varphi$, respectively. Recall the well-known fact that $\left\{M_{n}\right\}$ converges weakly to $M$ in $\mathcal{M}(\mathfrak{T}(\mathbb{G}))$ if and only if

$$
M(O) \leqq \liminf _{n \rightarrow \infty} M_{n}(O)
$$

holds for any open subset $O$ of $\mathfrak{T}(\mathbb{G})$. The topology on $\mathfrak{T}(\mathbb{G})$ yields that, for any open $O \subset \mathfrak{T}(\mathbb{G})$, there exists a disjoint family of cylindrical sets $\left\{O_{j}\right\}$ such that $O=\bigsqcup_{j} O_{j}$. Note that there are only a countable number of cylindrical sets. We 
have $M\left(O_{j}\right)=\lim _{n \rightarrow \infty} M_{n}\left(O_{j}\right)$ for $j$ by the assumption of convergence of $\varphi_{n}$ to $\varphi$. Then, (4.7) follows from

$$
M(O)=\sum_{j} M\left(O_{j}\right)=\sum_{j} \lim _{n \rightarrow \infty} M_{n}\left(O_{j}\right) \leqq \liminf _{n \rightarrow \infty} \sum_{j} M_{n}\left(O_{j}\right)=\liminf _{n \rightarrow \infty} M_{n}(O) .
$$

This completes the proof of continuity.

\section{REMARK 4.4}

The argument in the first paragraph of the above proof yields also that $\mathcal{M}(\mathfrak{T}(\mathbb{G}))$ is a closed subset of $\mathcal{P}(\mathfrak{T}(\mathbb{G}))$.

\section{LEMMA 4.5}

Assume that $\widehat{G}_{n}$ is at most countable for any $n \in \mathbb{N}$. The bijective map

$$
\mathcal{K}\left(G_{\infty}\right) \longrightarrow \mathcal{H}(\mathbb{G})
$$

given by the correspondence through (4.5) between $f \in \mathcal{K}\left(G_{\infty}\right)$ and $\varphi \in \mathcal{H}(\mathbb{G})$ is a homeomorphism.

Proof

Let a sequence $\left\{f_{n}\right\}$ converge to $f$ in $\mathcal{K}\left(G_{\infty}\right)$. Here, (4.5) implies that

$$
\varphi(\alpha)=\int_{G_{k}} f(x) \overline{\chi^{\alpha}(x)} d x, \quad \alpha \in \widehat{G}_{k},
$$

and also a similar equality for $f_{n}$ and the corresponding $\varphi_{n}$. Then we have for any $k \in \mathbb{N}$ and any $\alpha \in \widehat{G}_{k}$,

$$
\begin{aligned}
\left|\varphi_{n}(\alpha)-\varphi(\alpha)\right| & \leqq \int_{G_{k}}\left|f_{n}(x)-f(x)\right|\left|\chi^{\alpha}(x)\right| d x \\
& \leqq \operatorname{dim} \alpha \cdot \max _{x \in G_{k}}\left|f_{n}(x)-f(x)\right| \underset{n \rightarrow \infty}{\longrightarrow} 0 .
\end{aligned}
$$

This means convergence of $\left\{\varphi_{n}\right\}$ to $\varphi$ in $\mathcal{H}(\mathbb{G})$.

To show continuity of the inverse map, let a sequence $\left\{\varphi_{n}\right\}$ converge to $\varphi$ in $\mathcal{H}(\mathbb{G})$, noting that $\mathcal{H}(\mathbb{G})$ obviously enjoys the first countability since $\mathbb{G}$ has only a countable number of finite subsets. Let $k \in \mathbb{N}$ be fixed, and take an arbitrary $\epsilon>0$. Since

$$
\sum_{\alpha \in \widehat{G}_{k}} d(\alpha) \varphi(\alpha)=\varphi(\varnothing)=f(e)=1
$$

holds, there exists a finite subset $K_{1} \subset \widehat{G}_{k}$ such that

$$
0 \leqq \sum_{\alpha \in \widehat{G}_{k} \backslash K_{1}} d(\alpha) \varphi(\alpha)<\epsilon .
$$

Moreover, since we have

$$
\begin{aligned}
\sum_{\alpha \in \widehat{G}_{k} \backslash K_{1}} d(\alpha) \varphi_{n}(\alpha)=1-\sum_{\alpha \in K_{1}} d(\alpha) \varphi_{n}(\alpha) & \\
\underset{n \rightarrow \infty}{\longrightarrow} 1-\sum_{\alpha \in K_{1}} d(\alpha) \varphi(\alpha) & =\sum_{\alpha \in \widehat{G}_{k} \backslash K_{1}} d(\alpha) \varphi(\alpha),
\end{aligned}
$$


there exists $n_{1} \in \mathbb{N}$ such that

$$
n>n_{1} \Longrightarrow \sum_{\alpha \in \widehat{G}_{k} \backslash K_{1}} d(\alpha) \varphi_{n}(\alpha)<\epsilon .
$$

Then, letting $f_{n}, f \in \mathcal{K}\left(G_{\infty}\right)$ correspond to $\varphi_{n}, \varphi$, respectively, we have for $x \in G_{k}$ and $n>n_{1}$,

$$
\begin{aligned}
& \left|f_{n}(x)-f(x)\right| \\
& \leq\left|\sum_{\alpha \in \widehat{G}_{k} \backslash K_{1}} \varphi_{n}(\alpha) \chi^{\alpha}(x)\right|+\left|\sum_{\alpha \in \widehat{G}_{k} \backslash K_{1}} \varphi(\alpha) \chi^{\alpha}(x)\right| \\
& \quad+\left|\sum_{\alpha \in K_{1}}\left(\varphi_{n}(\alpha)-\varphi(\alpha)\right) \chi^{\alpha}(x)\right| \\
& \leqq \\
& \quad \sum_{\alpha \in \widehat{G}_{k} \backslash K_{1}} d(\alpha) \varphi_{n}(\alpha)+\sum_{\alpha \in \widehat{G}_{k} \backslash K_{1}} d(\alpha) \varphi(\alpha)+\sum_{\alpha \in K_{1}} d(\alpha)\left|\varphi_{n}(\alpha)-\varphi(\alpha)\right| \\
& <2 \epsilon+\sum_{\alpha \in K_{1}} d(\alpha)\left|\varphi_{n}(\alpha)-\varphi(\alpha)\right|,
\end{aligned}
$$

which yields

$$
\limsup _{n \rightarrow \infty} \max _{x \in G_{k}}\left|f_{n}(x)-f(x)\right| \leqq 2 \epsilon .
$$

Since $\epsilon>0$ is arbitrary, we have uniform convergence of $f_{n}$ to $f$ on $G_{k}$. Since $k \in \mathbb{N}$ is arbitrary, we have that $\lim _{n \rightarrow \infty} f_{n}=f$ in $\mathcal{K}\left(G_{\infty}\right)$.

Let us now return to the case of $G_{n}=\mathfrak{S}_{n}(T)$, the wreath product of compact group $T$ with at most countable $\widehat{T}$.

\section{THEOREM 4.6}

We have that $\operatorname{ex} \mathcal{H}(\mathbb{Y}(\widehat{T}))$, ex $\mathcal{M}(\mathfrak{T}(\widehat{T}))$, and $\operatorname{ex} \mathcal{K}\left(\mathfrak{S}_{\infty}(T)\right)$ are all homeomorphically isomorphic to $\Delta$ of (2.23). In particular, they are not compact if $T$ is infinite.

\section{Proof}

We showed in Lemmas 4.3 and 4.5 that the bijective maps between $\mathcal{H}(\mathbb{Y}(\widehat{T}))$, $\mathcal{M}(\mathfrak{T}(\widehat{T}))$, and $\mathcal{K}\left(\mathfrak{S}_{\infty}(T)\right)$ are all homeomorphisms. Since these maps are affine, they give homeomorphic isomorphisms between the sets of extremal points. By Theorem 3.3 we already know a bijective correspondence between ex $\mathcal{H}(\mathbb{Y}(\widehat{T}))$ and $\Delta$. Its homeomorphic property is read out through the explicit formula (2.28) for $K(\Lambda, \omega)$ in a similar argument to Step 2 of the proof of Theorem 2.5.

In contrast with Proposition 4.2, we consider the following question. 


\section{QUESTION 4.7}

Let the convergence of the normalized irreducible characters

$$
\lim _{n \rightarrow \infty} \tilde{\chi}^{\alpha^{(n)}}=f
$$

hold compact-uniformly on $G_{\infty}$ along a sequence $\left(\alpha^{(n)}\right)_{n=0,1,2, \ldots}, \alpha^{(n)} \in \widehat{G}_{n}$. Then, is the limit function $f$ a character of $G_{\infty}$ ?

Note that the limit on the left-hand side of (4.12) is well defined since every compact subset of $G_{\infty}$ is included in all sufficiently large $G_{n}$ 's. In the following statement, the Martin distance on $\mathbb{G}$ is given in a similar way to (1.29). (See also (A.16) in Appendix A.)

\section{LEMMA 4.8}

Assume that $\widehat{G}_{n}$ is at most countable for any $n \in \mathbb{N}$. For a sequence $\left(\alpha^{(n)}\right)_{n=0,1,2, \ldots}$, $\alpha^{(n)} \in \widehat{G}_{n}$, the following (a) and (b) are equivalent.

(a) There exists $\omega \in \partial \mathbb{G}$ such that

- $\alpha^{(n)}$ converges to $\omega$ as $n \rightarrow \infty$,

- $K(\cdot, \omega)$ is harmonic on $\mathbb{G}$.

(b) The normalized irreducible character $\widetilde{\chi}^{\alpha^{(n)}}$ converges compact-uniformly on $G_{\infty}$ as $n \rightarrow \infty$.

\section{Proof}

To deduce (b) from (a), we begin with

$$
\left.\widetilde{\chi}^{\alpha^{(n)}}\right|_{G_{k}}=\sum_{\alpha \in \widehat{G}_{k}} K\left(\alpha, \alpha^{(n)}\right) \chi^{\alpha} .
$$

The harmonicity of $K(\cdot, \omega)$ ensures that $f \in \mathcal{K}\left(G_{\infty}\right)$ is well defined by

$$
\left.f\right|_{G_{k}}=\sum_{\alpha \in \widehat{G}_{k}} K(\alpha, \omega) \chi^{\alpha} .
$$

In (4.13) and (4.14), (a) implies that $\lim _{n \rightarrow \infty} K\left(\alpha, \alpha^{(n)}\right)=K(\alpha, \omega)$ exists for any $\alpha \in \mathbb{G}$. Then, we estimate the supremum norm $\left\|f-\widetilde{\chi}^{\alpha^{(n)}}\right\|$ in the same way as (4.11). This yields (b).

Assuming (b), we have

$$
\lim _{n \rightarrow \infty} \widetilde{\chi}^{\alpha^{(n)}}=f \in \mathcal{K}\left(G_{\infty}\right)
$$

Take $\varphi \in \mathcal{H}(\mathbb{G})$ corresponding to $f$ via Proposition 4.1. Taking Fourier coefficient (4.9) and following (4.10), we see that

$$
K\left(\alpha, \alpha^{(n)}\right) \underset{n \rightarrow \infty}{\longrightarrow} \varphi(\alpha), \quad \alpha \in \mathbb{G} .
$$

Hence $\left(\alpha^{(n)}\right)$ converges to a point in the Martin boundary of $\mathbb{G}$, denoted by $\omega \in \partial \mathbb{G}$. Furthermore, $K(\cdot, \omega)=\varphi$ is harmonic. 


\section{REMARK 4.9}

In Lemma 4.8 , if the normalized irreducible characters $\widetilde{\chi}^{\alpha^{(n)}}$ are assumed to converge pointwise on $G_{\infty}$ instead of compact-uniformly, the limit function $f$ is still positive definite, central, and normalized. If we assume further that $f$ is continuous, then the convergence actually proves to be compact-uniform. Indeed, taking $\varphi \in \mathcal{H}(\mathbb{G})$ corresponding to $f \in \mathcal{K}\left(G_{\infty}\right)$, considering the Fourier coefficient as in (4.9), and then applying Lebesgue's convergence theorem, we have (4.15). It remains to repeat the estimate of (4.11) as in the first half of the proof of Lemma 4.8 .

The answer to Question 4.7 is affirmative for our wreath product case.

\section{PROPOSITION 4.10}

In the case of $G_{n}=\mathfrak{S}_{n}(T)$, Lemma $4.8(a)$ is equivalent to the following.

(a)' There exists $\omega \in \partial_{m} \mathbb{Y}(\widehat{T})$ such that $\alpha^{(n)}$ converges to $\omega$ as $n \rightarrow \infty$ (with respect to the Martin distance (1.29) on $\mathbb{Y}(\widehat{T})$ ).

Under (a), (a)', and/or (b), the limit function

$$
f=\lim _{n \rightarrow \infty} \widetilde{\chi}^{\alpha^{(n)}}
$$

is a character of $\mathfrak{S}_{\infty}(T)$. The correspondence $f \longleftrightarrow \omega$ is the one given in Theorems 3.3 and 4.6 .

Proof

We check that (a)' follows from apparently weak (a). Under (a), Lemma 2.8 ensures that $\omega \in \Delta$. Then, $\omega \in \partial_{m} \mathbb{Y}(\widehat{T})$ follows by Theorem 3.3.

\section{REMARK 4.11}

The answer to Question 4.7 is affirmative in the case of the infinite-dimensional unitary group $U(\infty)=\lim _{n \rightarrow \infty} U(n)$. (See [15, Proposition 10.9] and the references therein.) On the other hand, let each $G_{n}$ be a finite group in $G_{\infty}=$ $\lim _{n \rightarrow \infty} G_{n}$. In Lemma 4.8(a), the harmonicity of the Martin kernel $K(\cdot, \omega)$ then follows automatically from the other convergence condition. In [13, Section 1.5, Chapter 0], it is suggested that the $K(\cdot, \omega)$ obtained here is not necessarily extremal. This implies that the corresponding element in $\mathcal{K}\left(G_{\infty}\right)$, which is the limit function of $\widetilde{\chi}^{\alpha^{(n)}}$ in Lemma $4.8(\mathrm{~b})$, need not be a character of $G_{\infty}$.

\section{Appendix A: Markov chain and Martin boundary}

This appendix is devoted to a brief review on the Martin boundary associated with a Markov chain. Such a review is supplied because the definition (1.18) of harmonicity might seem to be strange from probabilistic viewpoints. As a main reference, we use [16], which is well written and most suitable for our purpose. Let $S$ be a countable set. A transition probability on $S$ is by definition a function 
$p(x, y)$ on $S \times S$ satisfying $p(x, y) \geqq 0(x, y \in S)$ and $\sum_{y \in S} p(x, y)=1(x \in S)$. Any transition probability induces a random motion on $S$, called a (temporally homogeneous) Markov chain on state space $S$, in which $p(x, y)$ is interpreted as the probability that the chain moves from $x$ to $y$ by one unit time. We have the transition matrix $P=[p(x, y)]_{x, y \in S}$ by giving $S$ a total order. For $n \in \mathbb{N} \cup\{0\}$ and $x, y \in S$, set

$$
\begin{aligned}
p_{n}(x, y) & =\left(P^{n}\right)_{x, y}=\sum_{z_{1}, \ldots, z_{n-1} \in S} p\left(x, z_{1}\right) p\left(z_{1}, z_{2}\right) \cdots p\left(z_{n-1}, y\right), \\
G_{p}(x, y) & =\sum_{n=0}^{\infty} p_{n}(x, y) .
\end{aligned}
$$

The subscript $p$ indicates dependence on the transition probability $p(x, y)$. Probability $\mathbb{P}$ on $S^{\infty}$ governing the Markov chain $\left(X_{n}\right)_{n=0,1,2, \ldots}$ is constructed via a well-known extension theorem so that

$$
p_{n}(x, y)=\mathbb{P}\left(X_{n}=y \mid X_{0}=x\right)
$$

holds. With $\mathbb{E}$ denoting the expectation with respect to $\mathbb{P}$,

$$
G_{p}(x, y)=\mathbb{E}\left[\sum_{n=0}^{\infty} 1_{\left\{X_{n}=y\right\}} \mid X_{0}=x\right]
$$

is the expected number for the chain to visit $y$ starting from $x$. Let us assume that a reference vertex $o$ is fixed and that

$$
G_{p}(o, y)>0, \quad y \in S .
$$

This is the case if the chain starting from $o$ can visit any vertex with positive probability. Set

$$
K_{p}(x, y)=\frac{G_{p}(x, y)}{G_{p}(o, y)}, \quad x, y \in S,
$$

and call it a Martin kernel. We define a distance on $S$ by

$$
D_{p}(x, y)=\sum_{z \in S} C(z)\left(\left|K_{p}(z, x)-K_{p}(z, y)\right|+\left|\delta_{z, x}-\delta_{z, y}\right|\right), \quad x, y \in S,
$$

where $C(z)$ is a positive coefficient for the sake of uniform convergence of the series in $x$ and $y$. In more detail, since we easily see that

$$
K_{p}(z, x) \leqq \frac{1}{p_{l}(o, z)}, \quad z, x \in S,
$$

by using $p_{l}(o, z)>0$ for some $l$, we only have to take $C(z)>0$ such that

$$
\sum_{z \in S} C(z)\left(\frac{2}{p_{l}(o, z)}+1\right)<\infty .
$$

We can then conclude that a sequence $\left\{x_{n}\right\}_{n \in \mathbb{N}}$ in $S$ is Cauchy if and only if either of the following holds:

(A.7) $x_{n} \equiv x \in S$ for sufficiently large $n$, 
(A.8) $\quad x_{n} \rightarrow \infty$ and, for any $z \in S,\left\{K_{p}\left(z, x_{n}\right)\right\}_{n \in \mathbb{N}}$ is a Cauchy sequence in $\mathbb{R}$. Moreover, any sequence in $S$ contains a Cauchy subsequence with respect to $D_{p}$, as seen from property (A.6) and the so-called diagonal argument. This implies that $S$ is totally bounded. The completion of $S$ with respect to $D_{p}$ is hence compact, which is called the Martin compactification of $S$ and is denoted by $\bar{S}$. The construction based on (A.5) ensures that $S$ is an open subset of $\bar{S}$ and that the restriction of the topology on $\bar{S}$ to $S$ is discrete. The compact set $\bar{S} \backslash S=\partial S$ is the Martin boundary of $S$. Martin kernel (A.4) is extended as a continuous function $K_{p}(x, \omega)$ on $S \times \bar{S}$.

Let us now assume that the nonnegative function $q(x, y)$ on $S \times S$ is given instead of a transition probability. Provided that there exists a positive $q$-harmonic function $h$ on $S$, that is,

$$
h(x)>0, \quad h(x)=\sum_{y \in S} q(x, y) h(y), \quad x \in S,
$$

we set a transition probability on $S$ by

$$
p(x, y)=\frac{1}{h(x)} q(x, y) h(y), \quad x, y \in S .
$$

Consider the analogue of (A.1) and (A.2) for $q(x, y)$ :

$$
\begin{aligned}
q_{n}(x, y) & =\sum_{z_{1}, \ldots, z_{n-1} \in S} q\left(x, z_{1}\right) q\left(z_{1}, z_{2}\right) \cdots q\left(z_{n-1}, y\right), \\
G_{q}(x, y) & =\sum_{n=0}^{\infty} q_{n}(x, y) .
\end{aligned}
$$

Comparing (A.11) with (A.2), we have

$$
G_{p}(x, y)=\frac{1}{h(x)} G_{q}(x, y) h(y), \quad x, y \in S .
$$

In particular, (A.3) is equivalent to the analogous condition

$$
G_{q}(o, y)>0, \quad y \in S .
$$

Furthermore, defining a Martin kernel by

$$
K_{q}(x, y)=\frac{G_{q}(x, y)}{G_{q}(o, y)}, \quad x, y \in S,
$$

we have

$$
K_{p}(x, y)=\frac{h(o)}{h(x)} K_{q}(x, y), \quad x, y \in S
$$

Set now as in (A.5)

$$
D_{q}(x, y)=\sum_{z \in S} C^{\prime}(z)\left(\left|K_{q}(z, x)-K_{q}(z, y)\right|+\left|\delta_{z, x}-\delta_{z, y}\right|\right), \quad x, y \in S
$$

with positive factor $C^{\prime}(z)$ so that the series can converge uniformly in $x, y$. Obviously, (A.15) yields that the Cauchy property of a sequence in $S$ is equivalent 
with respect to either $D_{p}$ or $D_{q}$. We thus arrive at the same Martin boundary theory starting from transition probability $p(x, y)$ or (nontransition probability) $q(x, y)$ related as (A.10). Note that the function $\varphi$ on $S$ is $p$-harmonic if and only if $h \varphi$ is $q$-harmonic.

Let us turn to the branching graph $\mathbb{Y}(\widehat{T})$. Based on (nontransition probability)

$$
q(\Lambda, \mathrm{M})=\left\{\begin{array}{ll}
\kappa(\Lambda, \mathrm{M}) & \text { if } \Lambda \nearrow \mathrm{M}, \\
0 & \text { otherwise, }
\end{array} \quad \Lambda, \mathrm{M} \in \mathbb{Y}(\widehat{T})\right.
$$

the Martin boundary of $\mathbb{Y}(\widehat{T})$ is constructed as above. In particular, taking $\varnothing$ as the reference vertex $o$, we have $G_{q}(\varnothing, \mathrm{M})=d(\mathrm{M})>0$ as (A.13). An example of a $q$-harmonic function in (A.9) is given by the special case of $\omega=(0,0, c) \in \Delta$ in (2.28), namely,

$$
h(\Lambda)=\prod_{\zeta \in \widehat{T}} \frac{c_{\zeta}^{\left|\lambda^{\zeta}\right|} \operatorname{dim} \lambda^{\zeta}}{(\operatorname{dim} \zeta)^{\left|\lambda^{\zeta}\right|}\left|\lambda^{\zeta}\right| !}, \quad \Lambda=\left(\lambda^{\zeta}\right)_{\zeta \in \widehat{T}} \in \mathbb{Y}(\widehat{T}) .
$$

It is straightforward to verify the $q$-harmonicity of (A.18) with respect to $q$ defined by (A.17), with $c_{\zeta}>0$ satisfying $\sum_{\zeta \in \widehat{T}} c_{\zeta}=1$. In the special case where $T$ is trivial, (A.18) reduces to

$$
h(\lambda)=\frac{\operatorname{dim} \lambda}{|\lambda| !}, \quad \lambda \in \mathbb{Y},
$$

and hence agrees with the harmonic function on $\mathbb{Y}$ corresponding to the Plancherel measure as well as the regular character of $\mathfrak{S}_{\infty}$. Applying (A.10) and (A.17) to (A.19), we have

$$
p(\lambda, \mu)=\frac{1}{n+1} \frac{\operatorname{dim} \mu}{\operatorname{dim} \lambda}, \quad \lambda \in \mathbb{Y}_{n} \nearrow \mu \in \mathbb{Y}_{n+1} .
$$

This induces a chain called the Plancherel growth process. If we take $h$ in (A.9) to be minimal $q$-harmonic (as is the case of (A.18) under (A.17)), then a bounded nonnegative $p$-harmonic function with respect to $p$ in (A.10) is necessarily constant, which follows from the minimality of $h$ as a $q$-harmonic function.

Martin kernel (A.14) is denoted by $K$ in (1.27). A straightforward translation of the discussion in this appendix leads to (1.29)-(1.34).

\section{References}

[1] A. Borodin and G. Olshanski, Harmonic functions on multiplicative graphs and interpolation polynomials, Electron. J. Combin. 7 (2000), no. 28. MR 1758654.

[2] R. Boyer, Character theory of infinite wreath products, Int. J. Math. Math. Sci. 9 (2005), 1365-1379. MR 2176493. DOI 10.1155/IJMMS.2005.1365.

[3] T. Hirai and E. Hirai, Positive definite class functions on a topological group and characters of factor representations, J. Math. Kyoto Univ. 45 (2005), 355-379. MR 2161697. 
[4] - "Character formula for wreath products of compact groups with the infinite symmetric group" in Quantum Probability, Banach Center Publ. 73, Polish Acad. Sci. Inst. Math., Warsaw, 2006, 207-221. MR 2423128. DOI 10.4064/bc73-0-15.

[5]_L Characters of wreath products of compact groups with the infinite symmetric group and characters of their canonical subgroups, J. Math. Kyoto Univ. 47 (2007), 269-320. MR 2376958.

[6] T. Hirai, E. Hirai, and A. Hora, Realizations of factor representations of finite type with emphasis on their characters for wreath products of compact groups with the infinite symmetric group, J. Math. Kyoto Univ. 46 (2006), 75-106. MR 2260818.

[7] Limits of characters of wreath products $\mathfrak{S}_{n}(T)$ of a compact group $T$ with the symmetric groups and characters of $\mathfrak{S}_{\infty}(T)$, I, Nagoya Math. J. 193 (2009), 1-93. MR 2502908.

[8] T. Hirai, H. Shimomura, N. Tatsuuma, and E. Hirai, Inductive limits of topologies, their direct products, and problems related to algebraic structures, J. Math. Kyoto Univ. 41 (2001), 475-505. MR 1878717.

[9] A. Hora, T. Hirai, and E. Hirai, Limits of characters of wreath products $\mathfrak{S}_{n}(T)$ of a compact group $T$ with the symmetric groups and characters of $\mathfrak{S}_{\infty}(T), I I$ : From a viewpoint of probability theory, J. Math. Soc. Japan 60 (2008), 1187-1217. MR 2467875.

[10] V. Ivanov and G. Olshanski, "Kerov's central limit theorem for the Plancherel measure on Young diagrams" in Symmetric Functions 2001: Surveys of Developments and Perspectives, NATO Sci. Ser. II Math. Phys. Chem. 74, Kluwer, Dordrecht, 2002, 93-151. MR 2059361. DOI 10.1007/978-94-010-0524-1_3.

[11] G. James and A. Kerber, The Representation Theory of the Symmetric Group, Encyclopedia Math. Appl. 16, Addison-Wesley, Reading, Mass., 1981. MR 0644144.

[12] S. Kerov, "The boundary of Young lattice and random Young tableaux" in Formal Power Series and Algebraic Combinatorics (New Brunswick, N.J., 1994, DIMACS Ser. Discrete Math. Theoret. Comput. Sci. 24, Amer. Math. Soc., Providence, 1996, 133-158. MR 1363510.

[13] - Asymptotic Representation Theory of the Symmetric Group and Its Applications in Analysis, Transl. Math. Monogr. 219, Amer. Math. Soc., Providence, 2003. MR 1984868.

[14] S. Kerov, A. Okounkov, and G. Olshanski, The boundary of the Young graph with Jack edge multiplicities, Int. Math. Res. Not. IMRN 1998, no. 4, 173-199. MR 1609628. DOI 10.1155/S1073792898000154.

[15] G. Olshanski, The problem of harmonic analysis on the infinite-dimensional unitary group, J. Funct. Anal. 205 (2003), 464-524. MR 2018416.

DOI 10.1016/S0022-1236(02)00022-8. 
[16] S. A. Sawyer, "Martin boundaries and random walks" in Harmonic Functions on Trees and Buildings (New York, 1995), Contemp. Math. 206, Amer. Math. Soc., Providence, 1997, 17-44. MR 1463727. DOI 10.1090/conm/206/02685.

[17] A. M. Vershik and S. V. Kerov, Asymptotic theory of the characters of the symmetric group (in Russian), Funktsional. Anal. i Prilozhen. 15, no. 4 (1981), 15-27; English translation in Funct. Anal. Appl. 15 (1982), 246-255. MR 0639197.

Hora: Department of Mathematics, Hokkaido University, Sapporo 060-0810, Japan; hora@math.sci.hokudai.ac.jp

Hirai: 22-8 Nakazaichi-Cho, Iwakura, Sakyo-Ku, Kyoto 606-0027, Japan; hira.takeshi.24e@st.kyoto-u.ac.jp 